\title{
13. STABLE ISOTOPE CHRONOLOGY AND PALEOCEANOGRAPHIC HISTORY OF SITES 963 AND 964, EASTERN MEDITERRANEAN SEA ${ }^{1}$
}

\author{
Michael W. Howell,,${ }^{2,6}$ Robert C. Thunell, ${ }^{3}$ Enrico Di Stefano, ${ }^{4}$ Rodolfo Sprovieri, ${ }^{4}$ Eric J. Tappa,${ }^{3}$ and Tatsuhiko Sakamoto ${ }^{5}$
}

\begin{abstract}
Oxygen and carbon isotope measurements were performed on the planktonic foraminifer Globigerina bulloides from Ocean Drilling Program Site 963 in the Strait of Sicily and Site 964 in the Ionian Sea. Isotope records from both sites reflect regional climate changes in the Mediterranean superimposed on a global climatic signal. The early to late Pleistocene $\delta^{18} \mathrm{O}$ record of Site 963 indicates that major climatic coolings occurred at approximately 0.98 and $0.45 \mathrm{Ma}$. The Site $964 \delta^{18} \mathrm{O}$ record extends into the early Pliocene and indicates that significant decreases in temperature and/or global ice volume occurred at 2.6, 0.98, and $0.46 \mathrm{Ma}$. Oxygen isotope records from both sites exhibit large amplitude fluctuations during the late Pleistocene associated with the reduction of surface-water salinities because of regional changes in evaporation and precipitation. The magnitude of these regional climate events appears to have been strongly influenced by the extent of global cooling and increases in ice volume. Carbon isotope records from both sites suggest (1) increased input of terrestrial organic matter, (2) higher nutrient concentrations within the photic zone, and (3) intensified surface-water stratification during the formation of sapropels. Data from both sites indicate no difference in the frequency of the surface-water salinity reductions, despite the fact that the deeper site (Site 964) exhibits a higher frequency of sapropels. This suggests that the reduction of surface-water salinities in the Strait of Sicily may have played a different role in the formation of sapropels at that site.
\end{abstract}

\section{INTRODUCTION}

Many Mediterranean paleoceanographic studies have focused on the formation of sapropels. These laminated sediments are usually rich in organic matter and generally are believed to have formed under anoxic or reducing conditions (Bradley, 1938; Olausson, 1961; Vergnaud-Grazzini et al., 1977; Calvert et al., 1992; Rossignol-Strick et al., 1982; among others). Most sapropel studies have focused on the Eastern Mediterranean Basin (e.g., Olausson, 1961; VergnaudGrazzini et al., 1977; Cita and Grignani 1982; Thunell et al., 1983; Calvert, 1983; Anastasakis and Stanley, 1986; Howell and Thunell, 1992) and units found in land-based sections (e.g., Van der Zwaan and Gudjonsson, 1986; Sprovieri et al., 1986; Howell et al., 1990; Hilgen, 1991; Lourens et al., 1992; Van Os et al., 1994), although sapropels have also been studied in the Western Mediterranean (Kastens, Mascle, Auroux, et al., 1987).

A key goal of most sapropel studies is to understand the mechanism by which these distinctive sediments formed. Many workers have attributed the formation of Eastern Mediterranean sapropels to the development of anoxic conditions in the Mediterranean, as a result of changes in basin hydrography (Olausson 1961; RossignolStrick et al., 1982; Thunell et al., 1983; Sarmiento et al., 1988; among others). The current circulation pattern in the Mediterranean can be described as anti-estuarine, where because of excess evaporation over precipitation, surface water from the North Atlantic flows in an eastward direction with a westward return flow at depth (Wüst, 1961; Béthoux, 1979). The deep water in the Eastern Mediterranean is formed north of the Levantine Basin and in the Adriatic Sea (Béthoux, 1989), and a cessation of deep-water production in these

${ }^{1}$ Robertson, A.H.F., Emeis, K.-C., Richter, C., and Camerlenghi, A. (Eds.), 1998. Proc. ODP, Sci. Results, 160: College Station, TX (Ocean Drilling Program).

${ }^{2}$ SCAMP and Marine Science Program, University of South Carolina, Columbia, SC 29208, U.S.A. howell@psc.sc.edu

${ }^{3}$ Department of Geological Sciences, University of South Carolina, Columbia, SC 29208, U.S.A.

${ }_{4}^{4}$ Dipartimento di Geologia e Geodesia, Università di Palermo, Corso Tukory, 13190134 Palermo, Italy.

${ }^{5}$ Division of Earth and Planetary Sciences, Graduate School of Science, Hokkaido University, Sapporo, 060, Japan.

${ }^{6}$ Present address: Department of Geological Sciences, University of South Carolina, Columbia, SC 29208, U.S.A. howell@epoch.geol.sc.edu regions could be the primary factor in developing bottom-water anoxia and the formation of sapropels. Distinctive "anomalies" in oxygen isotope signatures of planktonic foraminifers from sapropels (Emiliani, 1955, 1974; Stanley et al., 1975; Vergnaud-Grazzini et al., 1986; Rossignol-Strick et al., 1982; Ganssen and Troelstra, 1987; Thunell et al., 1987; Sarmiento et al., 1988; Howell and Thunell, 1992; among others) have been used to conclude that sapropels were formed during periods of reduced surface-water salinities, at times when the Mediterranean water balance may have been considerably different than today. An estuarine water balance may have inhibited deep-water formation by preventing the oxygen-rich waters in the northern parts of the Eastern Mediterranean from sinking. However, others have questioned the feasibility of an estuarine circulation pattern in the Mediterranean and provide alternative models to explain the formation of sapropels (e.g., Rohling and Gieskes, 1989; Rohling, 1991).

Alternatively, many workers have attributed the formation of Mediterranean sapropels to enhanced productivity (Calvert, 1983; Calvert et al., 1992; Howell and Thunell, 1992; among others). Under this scenario, increased surface-water eutrophication leads to the formation of reducing conditions, and enhanced organic matter preservation is the result of oxygen consumption rates exceeding renewal rates. Therefore, a key to understanding the origin of sapropels lies in understanding the hydrographic changes that occurred during their formation. Ocean Drilling Program (ODP) Leg 160 provided an opportunity to recover a transect of continuous sapropel-bearing cores from the Eastern Mediterranean that would encompass major portions of the Pliocene and Pleistocene. Previous Deep Sea Drilling Project (DSDP) efforts (Ryan, Hsü, et al., 1973; Hsü, Montadert, et al., 1978) were limited in terms of recovering pre-Pleistocene sediments from the Eastern Mediterranean. In addition, Leg 160 also provided the opportunity to integrate paleontological, isotopic, geochemical, and sedimentological studies that would facilitate a deeper understanding of the mechanism by which sapropels formed from spatial and temporal perspectives.

To achieve these objectives, information on temporal and spatial variations in salinity and sea-surface temperatures is necessary, as is a high-resolution stratigraphy. Isotope stratigraphy provides one approach through which these objectives can be met, as this technique has provided much information on the formation of sapropels in rela- 
tion to Mediterranean hydrography and climate. In this paper, we present the preliminary results of stable isotope analyses of planktonic foraminifers from Leg 160 Sites 963 and 964.

\section{Geological Setting}

Site 963 is located in the Strait of Sicily (Fig. 1), on a low ridge between the Gela Basin and Adventure Bank (Emeis, Robertson, Richter, et al., 1996). This ridge contains thick Pliocene-Pleistocene deposits from a small series of intrashelf basins. At $\sim 470 \mathrm{~m}$ water depth, Site 963 represents the shallowest of the sites drilled during Leg 160 and is located in a region of both compressional and extensional tectonism (see Emeis, Robertson, Richter, et al., 1996, for a complete description). Despite this, $\sim 474 \mathrm{~m}$ of relatively continuous Pliocene-Pleistocene section was recovered from this site.

The westward-flowing bottom waters in the Strait of Sicily come primarily from the Mediterranean Intermediate Water (MIW), which forms from the sinking of dense surface waters (Béthoux, 1989). Changes in surface-water hydrography in the Mediterranean during sapropel formation may have had an impact on the formation of the MIW. Stable isotope studies of planktonic foraminifers from this site should provide insight into the temporal variations in the surface waters at this location. Site 963 was also selected for the objective of extending the Pliocene and lower Pleistocene land-based paleoceanographic and climatic records (e.g., Rio et al., 1984; Thunell et al., 1985; Sprovieri et al., 1986; Van der Zwaan and Gudjonsson, 1986) into the upper Pleistocene.

Site 964 is situated on the Pisano Plateau, at the foot of the Calabrian Ridge in the Ionian Abyssal Plain (Fig. 1). It is located at a water depth of $3650 \mathrm{~m}$ on a small ridge of the South Calabrian Ridge $\sim 200 \mathrm{~m}$ above the Ionian Abyssal Plain (Emeis, Robertson, Richter, et al., 1996). This site represents the deepest in a transect of sites drilled during Leg 160 for testing and evaluating various theories of sapropel formation. If sapropel formation depends primarily on the development and establishment of bottom-water anoxia, sapropel formation in the deeper basins of the Eastern Mediterranean should precede the formation of these sediments in shallower settings. Testing this hypothesis requires a high-resolution stratigraphic framework to constrain the timing of sapropel events at this site and to facilitate correlation with sapropels from other sites and those studied in Mediterranean land-based sections. The development of a detailed isotope stratigraphy will be an important step toward achieving this goal.

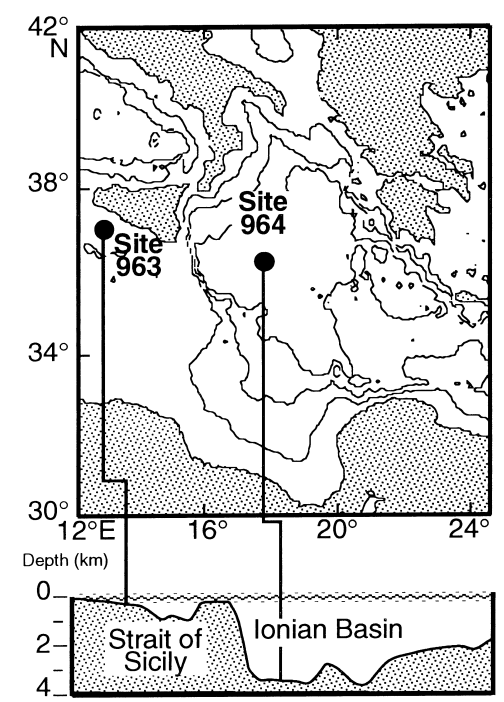

Figure 1. Location of Sites 963 and 964, Mediterranean Sea.

\section{METHODS}

To facilitate the recovery of a complete sedimentary sequence, multiple holes were hydraulically piston cored at Sites 963 and 964. A meter composite depth (mcd) scale was established to eliminate coring-induced gaps and overlaps in the sedimentary record and to facilitate correlation among cores from different holes for each site on the basis of gamma-ray attenuation porosity evaluator (GRAPE) analyses, magnetic susceptibility, and color reflectance data (Emeis, Robertson, Richter, et al., 1996). Cores from Sites 963 and 964 were sampled utilizing the splice tie points outlined in Emeis, Robertson, Richter, et al. (1996) to obtain a complete composite sequence. A revised mcd (rmcd) scale for Site 964 was developed by Sakamoto et al. (Chap. 4, this volume), and our sample depths for this site have been adjusted to this scale. A rmcd model for Site 963 is still under development, and the shipboard mcd scale is used for depth assignments in this report.

Cores from holes drilled at Sites 963 and 964 were sampled at 20$\mathrm{cm}$ intervals. Because of its high sedimentation rate (over $200 \mathrm{~m}$ in 1.5 m.y.), Site 963 samples were analyzed at 40-cm ( 3000 yr) intervals in the interest of time and resources. Samples from Site 964 were analyzed at $20-\mathrm{cm}$ intervals. Samples were disaggregated and specimens of the planktonic foraminifer Globigerina bulloides were isolated from washed residues for isotopic analysis. Picked specimens were sonically treated in methanol for $2 \mathrm{~min}$ before 5-10 individuals were picked for each analysis. All samples were analyzed at the University of South Carolina Stable Isotope Laboratory using a VG OPTIMA stable isotope ratio mass spectrometer equipped with an Isocarb preparation system. All stable isotope values are reported as per mil units $(\% \circ)$ relative to the PDB standard in $\delta$ notation. The standard error of reproducibility for all samples was $<0.05 \%$, with a standard analyzed for every 15 samples.

\section{Stratigraphic Control}

Table 1 provides a listing of the depths and ages of the calcareous nannofossil and paleomagnetic events identified at Site 963. The magnetostratigraphic framework described in Emeis, Robertson, Richter, et al. (1996) was utilized. Most of the Site 964 shipboard paleomagnetic record was deemed unsuitable for age determinations (see Emeis, Robertson, Richter, et al., 1996, for further discussion). Only the Brunhes/Matuyama boundary could be identified with confidence (A. Roberts, pers. comm., 1996) and was used for age control at this site (Table 2).

The stratigraphic position and ages of the biostratigraphic events are based on postcruise analyses by Di Stefano (Chap. 8, this volume) of samples from Hole 963B. Post-cruise efforts by Sprovieri et al. (Chap. 12, this volume) also provided a revised biostratigraphy for Site 964 that is used for age control in this study. The age assignments for the Pliocene and Pleistocene bioevents are reported in Table 2. We acknowledge that many of the age assignments used in this study are different from those reported by other workers (e.g., Castradori, 1993; Lourens et al., 1996). Additional work will be needed to assess the differences in the biostratigraphic chronologies. Age associations for samples from both sites were calculated through linear interpolation between the chronostratigraphic control points.

\section{SITE 963: OXYGEN ISOTOPES}

The results of the isotope analyses of $G$. bulloides for Site 963 are provided in Table 3 and are plotted against depth in Figure 2. Calcareous nannofossil and magnetic reversal stratigraphy indicates that the basal section of the analyzed composite is somewhere between 1.5 and 1.25 Ma. The long-term features of the Site 963 G. bulloides $\delta^{18} \mathrm{O}$ 
Table 1. Site 963 biostratigraphic and magnetostratigraphic events.

\begin{tabular}{lccl}
\hline \multicolumn{1}{c}{ Event } & $\begin{array}{c}\text { Depth } \\
(\mathrm{mcd})\end{array}$ & $\begin{array}{c}\text { Age } \\
(\mathrm{Ma})\end{array}$ & \multicolumn{1}{c}{ Source } \\
\hline Increase E. huxleyi & 32.19 & 0.050 & Castradori (1993) \\
FO E. huxleyi & 88.31 & 0.260 & Rio et al. (1990) \\
LO P. lacunosa & 118.6 & 0.460 & Rio et al. (1990) \\
LO Gephyrocapsa sp. 3 & 131.02 & 0.584 & Castradori (1993) \\
Brunhes/Matuyama & 149.23 & 0.780 & Cande and Kent (1995) \\
Bottom C1r.1 & 164.39 & 0.980 & Cande and Kent (1995) \\
FO Gephyrocapsa sp. 3 & 167.22 & 0.990 & Sprovieri (1993) \\
Bottom C1r.1n & 171.36 & 1.070 & Cande and Kent (1995) \\
LO Gephyrocapsa sp. $>5.5$ & 189.34 & 1.250 & Sprovieri (1993) \\
FO Gephyrocapsa sp. $>5.5$ & 210.83 & 1.500 & Sprovieri (1993) \\
& & & \\
& & &
\end{tabular}

Notes: Nannofossil data from postcruise study by Di Stefano (Chap. 8, this volume) of Hole 963B. FO = first occurrence. $\mathrm{LO}=$ last occurrence.

signal reflect major global climatic changes overprinted by regional climatic events. Fluctuations in the $\delta^{18} \mathrm{O}$ record of $G$. bulloides at Site 963 most likely reflect changes in temperature and global ice volume as a result of the expansion and contraction of Northern Hemisphere ice sheets.

To facilitate the chronological interpretation of this data, the $\delta^{18} \mathrm{O}$ record of Site 963 has been plotted against time (Fig. 4). We must stress that this age model is preliminary, and is provided only for the purpose of discussing the $\delta^{18} \mathrm{O}$ data within the context of a chronological framework. A more sophisticated age model will be the subject of a forthcoming paper. From the bottom of the studied section to the bottom of the $\mathrm{C} 1 \mathrm{r} .1$ magnetic event $(0.98 \mathrm{Ma}) \delta^{18} \mathrm{O}$ values for G. bulloides average $1.6 \%$ (Table 3 ). This interval includes a mixture of moderate to low-amplitude fluctuations up to $178 \mathrm{mcd}$, where a gradual decrease in $\delta^{18} \mathrm{O}$ occurs until $165 \mathrm{mcd}$. The highest frequencies of sapropels occurs within this interval (Fig. 4). Between 1.4 and $0.98 \mathrm{Ma}$, the Mediterranean and the global ocean were under the influence of glacial-interglacial climate oscillations dominated primarily by variations in the Earth's 41,000-year obliquity cycle. However, the amplitude of the Site 963 signal during this period is higher (up to $1.5 \%$ ) than that reported in open-ocean records (e.g., Broecker, 1986) and may reflect local changes in evaporation and precipitation, in addition to global climate changes.

A nearly $3.0 \%$ increase in the $\delta^{18} \mathrm{O}$ of $G$. bulloides that occurs just after 0.98 Ma may reflect the intensification of Northern Hemisphere glaciation, which is reflected in $\delta^{18} \mathrm{O}$ increases in global ocean records at this time (Shackleton and Opdyke, 1976; Ruddiman et al., 1986; Williams et al., 1988). Average $\delta^{18} \mathrm{O}$ values for $G$. bulloides increase by $\sim 0.22 \%$ and the amplitude of the signal also becomes more pronounced, as indicated by the increase in the average deviation of $\delta^{18} \mathrm{O}$ values from the mean (Table 4$)$.

Another major increase in oxygen isotope values occurs at $\sim 114$ mcd, between the extinction of the calcareous nannofossil Pseudoemiliana lacunosa $(0.46 \mathrm{Ma})$ and the top of the section $(0.038 \mathrm{Ma})$, where the $\delta^{18} \mathrm{O}$ signal increases by an average of $0.4 \%$ over the previous interval (Table 4). According to Ruddiman and Raymo (1988) the power of the 100-k.y. eccentricity cycle in global climate records culminated in a very strong signal at this time, which resulted in a more pronounced contrast between glacial and interglacial seasurface temperatures. We interpret the increase to reflect the response of the Strait of Sicily to this global climatic event. Similar observations have been documented by Thunell et al. (1990) at ODP Site 653 in the Tyrrhenian Sea. The Site $963 \delta^{18} \mathrm{O}$ record contains glacialinterglacial fluctuations of up to $3.3 \%$, which are considerably larger than the typical $1.5 \%$ glacial-interglacial changes in open-ocean records during this time period (e.g., Shackleton and Opdyke, 1976; Broecker, 1986). We interpret the high amplitude of this isotope record to reflect overprinting of the global climatic signal through the reduction of surface-water salinities in the Strait of Sicily brought on by regional changes in precipitation and evaporation. Similar observations have been made by other workers in studies of Eastern Mediterranean sapropels deposited during this time (Vergnaud-Grazzini et al., 1977, 1986; Williams et al., 1978; Williams and Thunell, 1979; Ganssen and Troelstra, 1987; Thunell et. al., 1990; among others). This indicates that the reduction of surface-water salinities was not restricted to the Eastern Mediterranean, and therefore the $\delta^{18} \mathrm{O}$ record of $G$. bulloides from the Strait of Sicily can provide an important record of global and regional climatic changes.

\section{SITE 964: OXYGEN ISOTOPES}

The results of the $\delta^{18} \mathrm{O}$ analyses of $G$. bulloides for Site 964 are given in Table 5 and are plotted against depth in Figure 3. Identification of the isotope stages is based on the biostratigraphy of Sprovieri et al. (Chap. 12, this volume). There are several intervals where data gaps exist in the isotope record because of an insufficient number of specimens of $G$. bulloides for analysis. The oxygen isotope record of G. bulloides for this site exhibits the characteristic long-term enrichment in $\delta^{18} \mathrm{O}$ values associated with the establishment and intensification of cooler climatic conditions and glaciation during the Pliocene/Pleistocene within the Mediterranean region (Keigwin and Thunell, 1979; Thunell and Williams, 1983; Thunell et al., 1990; Vergnaud-Grazzini et al., 1990) and glaciation during the PliocenePleistocene (Shackleton and Opdyke, 1976; Ruddiman et al., 1987; Shackleton et al., 1995).

To facilitate the chronological interpretation of these data, the $\delta^{18} \mathrm{O}$ record of Site 964 was plotted against time (Fig. 5). This preliminary age model is provided only for the discussion of the $\delta^{18} \mathrm{O}$ data within the context of a chronological framework, and a more sophisticated age model will be the subject of a forthcoming paper. Table 6 provides a summary of the average values and deviations from the mean in the $\delta^{18} \mathrm{O}$ record for selected intervals. To remove bias, values lower than $1.0 \%$ from sapropel samples have been omitted in the calculating mean $\delta^{18} \mathrm{O}$ values for samples younger than $1.5 \mathrm{Ma}$. Between the lowermost part of the record and 3.6 Ma, the mean $\delta^{18} \mathrm{O}$ of G. bulloides is $\sim 0.92 \%$ o, with relatively low-amplitude fluctuations. However, these estimates may be an artifact of the relatively coarse sampling resolution within this time interval. Between 3.5 and 3.2 $\mathrm{Ma}$, the mean $\delta^{18} \mathrm{O}$ values of $G$. bulloides decrease to $0.82 \%$. Mediterranean climate at this time has been characterized as warm (Thunell et al., 1990), as indicated by relatively low $\delta^{18} \mathrm{O}$ values. Therefore, the $\delta^{18} \mathrm{O}$ trends most likely reflect seasonal contrasts in temperature and changes in the overall balance of precipitation and evaporation in the Eastern Mediterranean. This interval of the Site 964 record may reflect both, and additional information (e.g., palynological data) is required to confirm this.

The $\delta^{18} \mathrm{O}$ values of $G$. bulloides increase by $1.5 \%$ o between 3.2 and $2.6 \mathrm{Ma}$ (Fig. 5), with the mean values for $\delta^{18} \mathrm{O}$ increasing by $0.25 \%$ (Table 6). In addition, the amplitude of the isotope signal is almost double that of the previous interval (Table 6). This may reflect increased cooling in this region of the Eastern Mediterranean accompanied by intensified contrasts in seasonal humidity. This may explain why glacial $\delta^{18} \mathrm{O}$ values at Site 964 are $\sim 0.4 \%$ o higher during this time period, in comparison to global records (e.g., Tiedemann et al., 1994; Shackleton et al., 1995). At 2.6 Ma, a short-term increase of almost $2 \%$ occurs in $\delta^{18} \mathrm{O}$ values of $G$. bulloides, and the mean values increase by an average of $0.4 \%$ over the preceding interval (Table 6). We attribute these changes in $\delta^{18} \mathrm{O}$ to a major increase in global ice volume resulting from intensified Northern Hemisphere glaciation. Faunal (Ciaranfi and Cita, 1973; Thunell, 1979), palynological (Zagwin, 1974; Suc, 1984, 1986) and isotopic (Thunell et al., 1990; Vergnaud Grazzini et al., 1990) studies have documented a major cooling event in the Mediterranean at this time. We conclude that the cooling 
Table 2. Site 964 biostratigraphic and magnetostratigraphic events.

\begin{tabular}{lrrl}
\hline \multicolumn{1}{c}{ Event } & $\begin{array}{r}\text { Depth } \\
\text { (rmcd) }\end{array}$ & $\begin{array}{c}\text { Age } \\
\text { (Ma) }\end{array}$ & \multicolumn{1}{c}{ Source } \\
\hline Increase E. huxleyi & 3.90 & 0.050 & Castradori (1993) \\
FO E. huxleyi & 14.24 & 0.260 & Rio et al. (1990) \\
LO P. lacunosa & 21.54 & 0.460 & Rio et al. (1990) \\
LO Gephyrocapsa sp. 3 & 24.86 & 0.584 & Castradori (1993) \\
Brunhes/Matuyama & 29.44 & 0.780 & Cande and Kent (1995) \\
FO Gephyrocapsa sp. 3 & 34.52 & 0.990 & Sprovieri (1993) \\
LO Gephyrocapsa. $>$ 5.5/ L.O. H. sellii & 40.71 & 1.250 & Sprovieri (1993) \\
FO Gephryocapsa $>$ 5.5 & 49.72 & 1.500 & Sprovieri (1993) \\
LO C. macintyrei & 54.92 & 1.630 & Sprovieri et al. (this volume) \\
FO medium size Gephyrocapsa & 57.12 & 1.750 & Sprovieri (1993) \\
Left N. pachyderma increase & 58.12 & 1.810 & Sprovieri (1993) \\
LO D. brouweri & 61.30 & 1.950 & Sprovieri et al. (this volume) \\
FO G. truncatulinoides & 64.90 & 2.070 & Sprovieri (1993) \\
FO G. inflata & 66.86 & 2.130 & Sprovieri (1993) \\
LO G. bononiensis & 82.61 & 2.450 & Sprovieri (1993) \\
LO D. pentaradiatus & 84.74 & 2.510 & Sprovieri (1993) \\
LO D. surculus & 85.54 & 2.530 & Sprovieri et al. (this volume) \\
LCO D. tamalis & 98.38 & 2.820 & Sprovieri (1993) \\
FO N. atlantica & 98.82 & 2.830 & Sprovieri (1993) \\
LO Sphaeroidinellopsis spp. & 109.92 & 3.220 & Sprovieri (1993) \\
LO G. puncticulata & 112.24 & 3.570 & Sprovieri (1993) \\
LO R. pseudoumbilicus & 116.39 & 3.850 & Sprovieri (1993)
\end{tabular}

Notes: Biostratigraphic data from postcruise study by Sprovieri et al. (Chap. 12, this volume) of Site 964. FO = first occurrence. $\mathrm{LO}=$ last occurrence.

Table 3. Site 963 stable isotope data (G. bulloides).

\begin{tabular}{|c|c|c|c|}
\hline Hole, core, section & $\begin{array}{l}\text { Depth } \\
\text { (mcd) }\end{array}$ & $\begin{array}{c}\delta^{18} \mathrm{O} \\
(\% o, \mathrm{PDB})\end{array}$ & $\begin{array}{c}\delta^{13} \mathrm{C} \\
(\% o, \text { PDB })\end{array}$ \\
\hline $963 \mathrm{~B}-1 \mathrm{H}-3$ & 4.47 & 2.180 & -0.933 \\
\hline $963 \mathrm{~B}-1 \mathrm{H}-4$ & 4.80 & 3.499 & -0.637 \\
\hline $963 \mathrm{~B}-1 \mathrm{H}-4$ & 5.20 & 2.825 & -1.086 \\
\hline $963 \mathrm{~B}-1 \mathrm{H}-4$ & 5.59 & 3.083 & -0.926 \\
\hline $963 \mathrm{~B}-1 \mathrm{H}-5$ & 6.30 & 3.385 & -0.712 \\
\hline $963 \mathrm{~B}-1 \mathrm{H}-5$ & 6.70 & 2.836 & -1.331 \\
\hline $963 \mathrm{~A}-2 \mathrm{H}-2$ & 8.30 & 3.497 & -0.963 \\
\hline $963 \mathrm{~A}-2 \mathrm{H}-2$ & 8.70 & 3.051 & -1.014 \\
\hline $963 \mathrm{~A}-2 \mathrm{H}-3$ & 9.60 & 3.020 & -1.245 \\
\hline $963 \mathrm{~A}-2 \mathrm{H}-3$ & 10.00 & 3.320 & -0.962 \\
\hline $963 \mathrm{~A}-2 \mathrm{H}-3$ & 10.40 & 3.386 & -1.112 \\
\hline $963 \mathrm{~A}-2 \mathrm{H}-4$ & 11.70 & 3.081 & -0.980 \\
\hline $963 \mathrm{~A}-2 \mathrm{H}-4$ & 12.08 & 3.233 & -0.639 \\
\hline $963 \mathrm{~A}-2 \mathrm{H}-5$ & 12.40 & 3.219 & -1.478 \\
\hline $963 \mathrm{~B}-2 \mathrm{H}-4$ & 14.41 & 3.395 & -1.695 \\
\hline $963 \mathrm{~B}-2 \mathrm{H}-5$ & 15.91 & 2.836 & -1.224 \\
\hline $963 \mathrm{~A}-3 \mathrm{H}-2$ & 18.29 & 3.263 & -1.005 \\
\hline $963 \mathrm{~A}-3 \mathrm{H}-2$ & 18.69 & 2.981 & -0.691 \\
\hline $963 \mathrm{~A}-3 \mathrm{H}-3$ & 19.41 & 2.915 & -1.024 \\
\hline $963 \mathrm{~A}-3 \mathrm{H}-3$ & 19.79 & 2.773 & -0.314 \\
\hline $963 \mathrm{~A}-3 \mathrm{H}-3$ & 19.79 & 2.625 & -0.772 \\
\hline $963 \mathrm{~A}-3 \mathrm{H}-3$ & 20.19 & 3.188 & -1.110 \\
\hline $963 \mathrm{~A}-3 \mathrm{H}-3$ & 20.55 & 3.091 & -1.216 \\
\hline $963 \mathrm{~A}-3 \mathrm{H}-5$ & 22.39 & 3.324 & -0.855 \\
\hline $963 \mathrm{~B}-3 \mathrm{H}-4$ & 25.21 & 2.260 & -1.229 \\
\hline $963 \mathrm{~B}-3 \mathrm{H}-5$ & 25.99 & 2.459 & -0.828 \\
\hline $963 \mathrm{~B}-3 \mathrm{H}-5$ & 26.79 & 2.762 & -0.615 \\
\hline $963 \mathrm{~A}-4 \mathrm{H}-3$ & 29.76 & 2.781 & -1.249 \\
\hline $963 \mathrm{~A}-4 \mathrm{H}-3$ & 30.14 & 3.091 & -0.879 \\
\hline $963 \mathrm{~A}-4 \mathrm{H}-4$ & 30.48 & 3.588 & -0.689 \\
\hline $963 \mathrm{~A}-4 \mathrm{H}-4$ & 31.06 & 3.318 & -0.568 \\
\hline $963 \mathrm{~A}-4 \mathrm{H}-4$ & 31.48 & 3.452 & -0.882 \\
\hline $963 \mathrm{~A}-4 \mathrm{H}-5$ & 32.16 & 3.383 & -0.938 \\
\hline $963 \mathrm{~A}-4 \mathrm{H}-5$ & 33.14 & 3.456 & -0.786 \\
\hline $963 \mathrm{~A}-4 \mathrm{H}-6$ & 33.46 & 3.440 & -1.118 \\
\hline $963 \mathrm{~A}-4 \mathrm{H}-6$ & 34.06 & 3.101 & -0.938 \\
\hline $963 \mathrm{~A}-4 \mathrm{H}-6$ & 34.28 & 2.048 & -0.836 \\
\hline $963 \mathrm{~A}-5 \mathrm{H}-1$ & 36.77 & 2.592 & -0.554 \\
\hline $963 \mathrm{~B}-4 \mathrm{H}-5$ & 36.88 & 2.099 & -0.833 \\
\hline $963 \mathrm{~B}-4 \mathrm{H}-5$ & 37.27 & 2.239 & -0.386 \\
\hline $963 \mathrm{~A}-5 \mathrm{H}-2$ & 37.69 & 1.517 & -1.312 \\
\hline $963 \mathrm{~B}-4 \mathrm{H}-5$ & 38.10 & 1.797 & -0.997 \\
\hline $963 \mathrm{~B}-4 \mathrm{H}-6$ & 38.38 & 1.680 & -0.840 \\
\hline $963 \mathrm{~A}-5 \mathrm{H}-3$ & 39.57 & 2.068 & -0.898 \\
\hline $963 \mathrm{~A}-5 \mathrm{H}-3$ & 39.97 & 1.553 & -1.816 \\
\hline $963 \mathrm{~A}-5 \mathrm{H}-3$ & 40.37 & 1.387 & -1.409 \\
\hline $963 \mathrm{~A}-5 \mathrm{H}-4$ & 41.07 & 2.751 & -0.259 \\
\hline $963 \mathrm{~A}-5 \mathrm{H}-4$ & 41.47 & 2.526 & -1.558 \\
\hline $963 \mathrm{~A}-5 \mathrm{H}-4$ & 41.83 & 1.806 & -0.997 \\
\hline $963 \mathrm{~A}-5 \mathrm{H}-5$ & 42.96 & 2.228 & -1.126 \\
\hline $963 \mathrm{~A}-5 \mathrm{H}-5$ & 43.34 & 2.071 & -0.538 \\
\hline $963 \mathrm{~A}-5 \mathrm{H}-5$ & 43.34 & 2.132 & -0.900 \\
\hline $963 \mathrm{~B}-5 \mathrm{H}-4$ & 44.60 & 2.638 & -0.639 \\
\hline $963 \mathrm{~B}-5 \mathrm{H}-4$ & 44.98 & 1.499 & -0.885 \\
\hline $963 \mathrm{~B}-5 \mathrm{H}-4$ & 45.38 & 1.501 & -0.769 \\
\hline $963 \mathrm{~B}-5 \mathrm{H}-4$ & 45.78 & 1.221 & -0.604 \\
\hline
\end{tabular}

\begin{tabular}{|c|c|c|c|}
\hline Hole, core, section & $\begin{array}{l}\text { Depth } \\
\text { (mcd) }\end{array}$ & $\begin{array}{c}\delta^{18} \mathrm{O} \\
(\% o, \text { PDB })\end{array}$ & $\begin{array}{c}\delta^{13} \mathrm{C} \\
(\%, \mathrm{PDB})\end{array}$ \\
\hline $963 \mathrm{~A}-6 \mathrm{H}-1$ & 47.20 & 0.364 & -2.066 \\
\hline $963 \mathrm{~A}-6 \mathrm{H}-2$ & 48.70 & 1.248 & -0.652 \\
\hline $963 \mathrm{~A}-6 \mathrm{H}-2$ & 49.08 & 1.429 & -0.840 \\
\hline $963 \mathrm{~A}-6 \mathrm{H}-3$ & 49.38 & 1.887 & -1.452 \\
\hline $963 \mathrm{~A}-6 \mathrm{H}-3$ & 50.38 & 2.588 & -0.913 \\
\hline $963 \mathrm{~A}-6 \mathrm{H}-4$ & 51.08 & 2.638 & -1.135 \\
\hline $963 \mathrm{~A}-6 \mathrm{H}-4$ & 51.48 & 3.010 & -1.177 \\
\hline $963 \mathrm{~A}-6 \mathrm{H}-4$ & 51.88 & 2.924 & -1.269 \\
\hline $963 \mathrm{~A}-6 \mathrm{H}-5$ & 52.20 & 2.453 & -0.758 \\
\hline $963 \mathrm{~A}-6 \mathrm{H}-5$ & 52.58 & 3.313 & -1.054 \\
\hline $963 \mathrm{~A}-6 \mathrm{H}-5$ & 53.38 & 2.917 & -1.718 \\
\hline $963 \mathrm{~A}-7 \mathrm{H}-2$ & 58.97 & 1.483 & -1.346 \\
\hline $963 \mathrm{~A}-7 \mathrm{H}-3$ & 59.67 & 1.753 & -1.137 \\
\hline $963 \mathrm{~A}-7 \mathrm{H}-3$ & 60.07 & 1.805 & -0.987 \\
\hline $963 \mathrm{~A}-7 \mathrm{H}-3$ & 60.47 & 1.973 & -1.344 \\
\hline $963 \mathrm{~A}-7 \mathrm{H}-4$ & 61.17 & 2.199 & -1.186 \\
\hline $963 \mathrm{~A}-7 \mathrm{H}-4$ & 61.57 & 2.246 & -0.945 \\
\hline $963 \mathrm{~A}-7 \mathrm{H}-5$ & 62.67 & 1.304 & -2.087 \\
\hline $963 \mathrm{~B}-7 \mathrm{H}-4$ & 64.69 & 3.043 & -1.132 \\
\hline $963 \mathrm{~B}-7 \mathrm{H}-4$ & 64.69 & 3.078 & -1.137 \\
\hline $963 \mathrm{~B}-7 \mathrm{H}-4$ & 65.08 & 3.085 & -1.014 \\
\hline $963 \mathrm{~B}-7 \mathrm{H}-4$ & 65.49 & 2.312 & -1.200 \\
\hline $963 \mathrm{~A}-8 \mathrm{H}-1$ & 65.96 & 1.395 & -1.582 \\
\hline $963 \mathrm{~A}-8 \mathrm{H}-1$ & 66.40 & 0.413 & -1.860 \\
\hline $963 \mathrm{~A}-8 \mathrm{H}-2$ & 66.70 & 0.604 & -0.783 \\
\hline $963 \mathrm{~A}-8 \mathrm{H}-2$ & 67.08 & 0.435 & -0.982 \\
\hline $963 \mathrm{~A}-8 \mathrm{H}-2$ & 67.48 & 0.458 & -1.427 \\
\hline $963 \mathrm{~A}-8 \mathrm{H}-2$ & 67.90 & 0.098 & -1.566 \\
\hline $963 \mathrm{~A}-8 \mathrm{H}-3$ & 68.20 & -0.244 & -1.847 \\
\hline $963 \mathrm{~A}-8 \mathrm{H}-3$ & 68.60 & 1.469 & -1.507 \\
\hline $963 \mathrm{~A}-8 \mathrm{H}-3$ & 68.98 & 2.171 & -1.318 \\
\hline $963 \mathrm{~A}-8 \mathrm{H}-3$ & 69.40 & 2.042 & -1.276 \\
\hline $963 \mathrm{~A}-8 \mathrm{H}-4$ & 69.70 & 1.998 & -1.538 \\
\hline $963 \mathrm{~A}-8 \mathrm{H}-4$ & 70.10 & 2.044 & -1.029 \\
\hline $963 \mathrm{~A}-8 \mathrm{H}-4$ & 70.48 & 2.337 & -1.474 \\
\hline $963 \mathrm{~A}-8 \mathrm{H}-4$ & 70.90 & 1.280 & -1.362 \\
\hline $963 \mathrm{~A}-8 \mathrm{H}-5$ & 71.58 & 1.470 & -0.596 \\
\hline $963 \mathrm{~A}-8 \mathrm{H}-5$ & 72.40 & 2.394 & -0.366 \\
\hline $963 \mathrm{~A}-8 \mathrm{H}-6$ & 72.70 & 1.675 & -0.953 \\
\hline $963 \mathrm{~A}-9 \mathrm{H}-1$ & 74.70 & 1.657 & -1.251 \\
\hline $963 \mathrm{~A}-9 \mathrm{H}-1$ & 75.10 & -0.276 & -1.524 \\
\hline $963 \mathrm{~A}-9 \mathrm{H}-1$ & 75.50 & 0.190 & -1.709 \\
\hline $963 \mathrm{~A}-9 \mathrm{H}-1$ & 75.90 & 0.680 & -0.842 \\
\hline $963 \mathrm{~A}-9 \mathrm{H}-2$ & 76.20 & 0.331 & -1.075 \\
\hline $963 \mathrm{~A}-9 \mathrm{H}-2$ & 76.60 & 1.542 & -1.710 \\
\hline $963 \mathrm{~A}-9 \mathrm{H}-2$ & 77.00 & 1.254 & -1.549 \\
\hline $963 \mathrm{~A}-9 \mathrm{H}-2$ & 77.40 & 1.544 & -1.004 \\
\hline $963 \mathrm{~A}-9 \mathrm{H}-3$ & 77.70 & 1.626 & -1.478 \\
\hline $963 \mathrm{~A}-9 \mathrm{H}-3$ & 78.10 & 1.776 & -1.234 \\
\hline $963 \mathrm{~A}-9 \mathrm{H}-3$ & 78.90 & 1.042 & -1.719 \\
\hline $963 \mathrm{~A}-9 \mathrm{H}-4$ & 79.20 & 0.727 & -1.590 \\
\hline $963 \mathrm{~A}-9 \mathrm{H}-4$ & 80.00 & 1.438 & -0.641 \\
\hline $963 \mathrm{~A}-9 \mathrm{H}-4$ & 80.40 & 2.300 & -0.777 \\
\hline $963 \mathrm{~A}-9 \mathrm{H}-5$ & 80.70 & 2.409 & -0.822 \\
\hline 963A-9H-5 & 81.50 & 2.550 & -1.485 \\
\hline $963 \mathrm{~A}-9 \mathrm{H}-6$ & 82.20 & 2.466 & -1.339 \\
\hline
\end{tabular}


Table 3 (continued).

\begin{tabular}{|c|c|c|c|}
\hline Hole, core, section & $\begin{array}{l}\text { Depth } \\
\text { (mcd) }\end{array}$ & $\begin{array}{c}\delta^{18} \mathrm{O} \\
(\%, \mathrm{PDB})\end{array}$ & $\begin{array}{c}\delta^{13} \mathrm{C} \\
(\%, \mathrm{PDB})\end{array}$ \\
\hline $963 \mathrm{~A}-9 \mathrm{H}-1$ & 84.61 & 2.682 & -1.335 \\
\hline $963 \mathrm{~A}-9 \mathrm{H}-1$ & 85.00 & 2.688 & -1.445 \\
\hline 963B-9H-5 & 85.18 & 2.497 & -1.049 \\
\hline $963 \mathrm{~A}-9 \mathrm{H}-1$ & 85.40 & 2.886 & -1.505 \\
\hline $963 \mathrm{~B}-9 \mathrm{H}-5$ & 85.59 & 2.226 & -1.439 \\
\hline $963 \mathrm{~B}-9 \mathrm{H}-5$ & 85.59 & 2.302 & -1.412 \\
\hline $963 \mathrm{~A}-10-2$ & 85.70 & 2.389 & -1.317 \\
\hline $963 \mathrm{~A}-10-2$ & 86.10 & 3.164 & -1.367 \\
\hline $963 \mathrm{~A}-10-3$ & 87.18 & 3.214 & -1.617 \\
\hline $963 \mathrm{~A}-10-3$ & 87.58 & 3.193 & -1.484 \\
\hline $963 \mathrm{~A}-10-3$ & 87.99 & 3.337 & -1.587 \\
\hline $963 \mathrm{~A}-10-3$ & 88.39 & 3.191 & -1.383 \\
\hline $963 \mathrm{~A}-10-3$ & 88.39 & 3.171 & -1.410 \\
\hline $963 \mathrm{~A}-10-4$ & 89.49 & 2.501 & -0.780 \\
\hline $963 \mathrm{~B}-10-4$ & 90.29 & 1.573 & -1.212 \\
\hline $963 \mathrm{~B}-10-4$ & 90.68 & 1.624 & -0.878 \\
\hline $963 \mathrm{~B}-10-4$ & 91.48 & 2.168 & -1.649 \\
\hline $963 \mathrm{~B}-10-5$ & 92.64 & 2.920 & -1.162 \\
\hline $963 \mathrm{~B}-10-5$ & 92.98 & 2.650 & -1.136 \\
\hline $963 \mathrm{~B}-10-6$ & 93.29 & 2.057 & -1.174 \\
\hline $963 \mathrm{~B}-10-6$ & 93.67 & 1.375 & -1.750 \\
\hline $963 \mathrm{~A}-11-1$ & 94.09 & 1.294 & -1.742 \\
\hline $963 \mathrm{~B}-10-6$ & 94.14 & 2.083 & -1.011 \\
\hline $963 \mathrm{~B}-11-1$ & 95.89 & 1.265 & -0.554 \\
\hline $963 \mathrm{~B}-11-2$ & 96.57 & 0.824 & -1.421 \\
\hline $963 \mathrm{~B}-11-3$ & 98.07 & 0.534 & -1.239 \\
\hline $963 \mathrm{~B}-11-3$ & 98.89 & 1.535 & -2.210 \\
\hline $963 \mathrm{~B}-11-4$ & 99.57 & 2.572 & -1.575 \\
\hline $963 \mathrm{~B}-11-4$ & 99.98 & 3.436 & -1.245 \\
\hline $963 \mathrm{~B}-11-4$ & 100.39 & 3.003 & -1.447 \\
\hline $963 \mathrm{~B}-11-5$ & 101.48 & 3.392 & -0.839 \\
\hline $963 \mathrm{~B}-11-5$ & 101.89 & 3.006 & -1.119 \\
\hline $963 \mathrm{~A}-12-2$ & 104.69 & 2.408 & -0.715 \\
\hline $963 \mathrm{~A}-12-4$ & 106.90 & 1.433 & -0.905 \\
\hline $963 \mathrm{~A}-12-5$ & 108.40 & 0.943 & -0.632 \\
\hline $963 \mathrm{~A}-12-5$ & 109.19 & 1.038 & -1.074 \\
\hline $963 \mathrm{~A}-13-1$ & 111.78 & 1.373 & -1.148 \\
\hline $963 \mathrm{~A}-13-1$ & 112.18 & 1.912 & -1.275 \\
\hline $963 \mathrm{~A}-13-2$ & 113.28 & 2.376 & -0.592 \\
\hline $963 \mathrm{~A}-13-2$ & 113.68 & 2.405 & -1.085 \\
\hline $963 \mathrm{~A}-13-3$ & 114.58 & 1.293 & -1.639 \\
\hline $963 \mathrm{~A}-13-3$ & 114.98 & 2.257 & -1.148 \\
\hline $963 \mathrm{~A}-13-4$ & 117.08 & 2.050 & -0.483 \\
\hline $963 \mathrm{~A}-13-4$ & 117.48 & 1.972 & -0.758 \\
\hline $963 \mathrm{~A}-13-5$ & 117.78 & 2.977 & -1.067 \\
\hline $963 \mathrm{~A}-13-5$ & 118.18 & 3.137 & -0.386 \\
\hline $963 \mathrm{~A}-13-5$ & 118.58 & 1.896 & -0.989 \\
\hline $963 \mathrm{~A}-14-1$ & 118.60 & 1.958 & -1.339 \\
\hline $963 \mathrm{~A}-14-1$ & 119.00 & 2.249 & -0.655 \\
\hline $963 \mathrm{~A}-14-1$ & 119.40 & 2.113 & -0.031 \\
\hline $963 \mathrm{~A}-14-1$ & 119.80 & 1.367 & -1.029 \\
\hline $963 \mathrm{~A}-14-2$ & 120.10 & 1.209 & -0.811 \\
\hline $963 \mathrm{~A}-14-2$ & 120.50 & 1.263 & -0.976 \\
\hline $963 \mathrm{~A}-14-2$ & 120.90 & 1.401 & -0.838 \\
\hline $963 \mathrm{~A}-14-2$ & 121.30 & 1.103 & -1.034 \\
\hline $963 \mathrm{~A}-14-3$ & 121.60 & 1.594 & -0.713 \\
\hline $963 \mathrm{~A}-14-3$ & 122.00 & 1.273 & -0.937 \\
\hline $963 \mathrm{~A}-14-3$ & 122.40 & 1.009 & -1.552 \\
\hline $963 \mathrm{~A}-14-3$ & 122.80 & 0.948 & -1.688 \\
\hline $963 \mathrm{~A}-14-4$ & 123.10 & 1.411 & -1.225 \\
\hline $963 \mathrm{~A}-14-4$ & 123.50 & 2.236 & -0.853 \\
\hline $963 \mathrm{~A}-14-4$ & 123.90 & 1.915 & -1.107 \\
\hline $963 \mathrm{~A}-14-4$ & 124.30 & 1.551 & -0.867 \\
\hline $963 \mathrm{~A}-14-5$ & 124.60 & 1.540 & -0.576 \\
\hline $963 \mathrm{~B}-14-4$ & 124.90 & 1.661 & -0.522 \\
\hline $963 \mathrm{~B}-14-5$ & 126.62 & 2.448 & -1.132 \\
\hline $963 \mathrm{~B}-14-5$ & 127.00 & 0.855 & -1.405 \\
\hline $963 \mathrm{~A}-15-1$ & 128.51 & 1.326 & -1.439 \\
\hline $963 B-14-6$ & 128.71 & 1.577 & -0.759 \\
\hline $963 \mathrm{~A}-15-1$ & 128.91 & 2.161 & -1.025 \\
\hline $963 \mathrm{~A}-15-1$ & 129.31 & 1.938 & -0.778 \\
\hline $963 \mathrm{~A}-15-1$ & 129.71 & 0.792 & -1.616 \\
\hline $963 \mathrm{~A}-15-2$ & 130.01 & 0.756 & -1.500 \\
\hline $963 \mathrm{~A}-15-2$ & 130.81 & 1.719 & -0.819 \\
\hline $963 \mathrm{~A}-15-3$ & 131.51 & 1.367 & -1.362 \\
\hline $963 \mathrm{~A}-15-3$ & 132.31 & 1.833 & -0.908 \\
\hline $963 \mathrm{~A}-15-3$ & 132.71 & 1.809 & 0.085 \\
\hline $963 \mathrm{~A}-15-4$ & 133.01 & 0.648 & -1.724 \\
\hline $963 \mathrm{~A}-15-5$ & 134.51 & 1.098 & -0.867 \\
\hline $963 \mathrm{~A}-16-1$ & 137.51 & 2.742 & -1.089 \\
\hline $963 \mathrm{~A}-16-1$ & 138.71 & 2.045 & -1.559 \\
\hline $963 \mathrm{~A}-16-2$ & 139.41 & 2.644 & -1.673 \\
\hline $963 \mathrm{~A}-16-2$ & 139.82 & 2.117 & -1.428 \\
\hline $963 \mathrm{~A}-16-2$ & 140.21 & 1.877 & -1.496 \\
\hline $963 \mathrm{~A}-16-3$ & 140.51 & 2.306 & -1.212 \\
\hline $963 \mathrm{~A}-16-3$ & 140.91 & 1.274 & -1.931 \\
\hline $963 \mathrm{~A}-16-3$ & 141.32 & 1.742 & -0.851 \\
\hline $963 \mathrm{~A}-16-3$ & 141.71 & 0.894 & -1.389 \\
\hline $963 \mathrm{~A}-16-4$ & 142.01 & 1.085 & -1.032 \\
\hline $963 \mathrm{~B}-17-1$ & 146.83 & 2.621 & -0.876 \\
\hline
\end{tabular}

\begin{tabular}{|c|c|c|c|}
\hline Hole, core, section & $\begin{array}{l}\text { Depth } \\
\text { (mcd) }\end{array}$ & $\begin{array}{c}\delta^{18} \mathrm{O} \\
(\% o, \mathrm{PDB})\end{array}$ & $\begin{array}{c}\delta^{13} \mathrm{C} \\
(\%, \mathrm{PDB})\end{array}$ \\
\hline $963 \mathrm{~B}-17-2$ & 147.32 & 1.641 & -1.317 \\
\hline $963 \mathrm{~B}-17-2$ & 147.74 & 2.918 & -0.849 \\
\hline $963 \mathrm{~B}-17-2$ & 148.54 & 2.175 & -0.326 \\
\hline $963 \mathrm{~A}-17-2$ & 148.91 & 1.905 & -0.935 \\
\hline $963 \mathrm{~A}-17-2$ & 149.71 & 0.904 & -1.748 \\
\hline $963 \mathrm{~A}-17-3$ & 150.01 & 1.512 & -1.561 \\
\hline $963 \mathrm{~A}-17-3$ & 150.41 & 2.223 & -1.290 \\
\hline $963 \mathrm{~A}-17-3$ & 150.81 & 2.563 & -1.447 \\
\hline $963 \mathrm{~A}-17-3$ & 151.21 & 2.970 & -0.928 \\
\hline $963 \mathrm{~A}-17-4$ & 151.51 & 3.217 & -1.484 \\
\hline $963 \mathrm{~A}-17-4$ & 151.91 & 2.650 & -0.883 \\
\hline $963 \mathrm{~A}-17-4$ & 152.69 & 1.930 & -0.558 \\
\hline $963 \mathrm{~B}-18-1$ & 153.30 & 1.568 & -1.136 \\
\hline $963 \mathrm{~B}-18-1$ & 153.70 & 1.544 & -1.028 \\
\hline $963 \mathrm{~B}-18-2$ & 154.10 & 1.499 & -1.017 \\
\hline $963 \mathrm{~B}-18-2$ & 154.50 & 0.893 & -1.367 \\
\hline $963 \mathrm{~B}-18-2$ & 154.91 & 2.057 & -0.779 \\
\hline $963 \mathrm{~B}-18-4$ & 157.11 & 1.775 & -1.317 \\
\hline $963 \mathrm{~B}-18-4$ & 157.40 & 2.786 & -1.738 \\
\hline $963 \mathrm{~B}-18-4$ & 158.21 & 3.139 & -1.510 \\
\hline $963 \mathrm{~B}-18-5$ & 159.41 & 2.079 & -1.018 \\
\hline $963 \mathrm{~B}-18-6$ & 160.21 & 1.570 & -1.439 \\
\hline $963 \mathrm{~B}-18-6$ & 160.61 & 1.841 & -1.281 \\
\hline $963 \mathrm{~B}-18-6$ & 160.99 & 1.227 & -1.838 \\
\hline $963 \mathrm{~B}-18-6$ & 161.38 & 1.667 & -1.496 \\
\hline $963 \mathrm{~A}-19-2$ & 162.81 & 0.311 & -1.790 \\
\hline $963 \mathrm{~A}-19-3$ & 163.21 & 2.162 & -0.907 \\
\hline $963 \mathrm{~A}-19-3$ & 163.61 & 1.614 & -1.142 \\
\hline $963 \mathrm{~A}-19-3$ & 164.01 & 2.095 & -0.788 \\
\hline $963 \mathrm{~B}-19-2$ & 164.32 & 0.250 & -1.287 \\
\hline $963 \mathrm{~B}-19-2$ & 164.72 & 1.451 & -1.261 \\
\hline $963 \mathrm{~B}-19-3$ & 165.12 & 1.203 & -1.361 \\
\hline $963 \mathrm{~B}-19-3$ & 165.52 & 1.260 & -0.934 \\
\hline $963 \mathrm{~A}-20-2$ & 169.09 & 2.260 & -0.731 \\
\hline $963 \mathrm{~A}-20-3$ & 169.51 & 1.521 & -1.154 \\
\hline $963 \mathrm{~A}-20-3$ & 169.91 & 0.853 & -1.943 \\
\hline $963 \mathrm{~A}-20-3$ & 170.31 & 1.965 & -1.387 \\
\hline $963 \mathrm{~A}-20-3$ & 170.71 & 1.766 & -1.728 \\
\hline $963 \mathrm{~A}-20-4$ & 171.11 & 1.774 & -1.090 \\
\hline $963 \mathrm{~A}-20-5$ & 172.31 & 0.870 & -0.755 \\
\hline $963 \mathrm{~A}-20-5$ & 173.31 & 0.952 & -1.261 \\
\hline $963 \mathrm{~A}-21-1$ & 174.51 & 2.486 & -0.698 \\
\hline $963 \mathrm{~A}-21-1$ & 174.91 & 1.997 & -1.026 \\
\hline $963 \mathrm{~A}-21-1$ & 175.31 & 2.101 & -0.907 \\
\hline $963 \mathrm{~A}-21-1$ & 175.71 & 1.233 & -1.180 \\
\hline $963 \mathrm{~A}-21-2$ & 176.02 & 1.122 & -1.357 \\
\hline $963 \mathrm{~A}-21-3$ & 177.41 & 2.631 & -1.609 \\
\hline $963 \mathrm{~A}-21-3$ & 177.82 & 2.747 & -1.200 \\
\hline $963 \mathrm{~A}-21-3$ & 178.21 & 1.920 & -1.558 \\
\hline $963 \mathrm{~A}-21-3$ & 178.61 & 2.093 & -1.100 \\
\hline $963 \mathrm{~A}-21-4$ & 179.01 & 2.218 & -1.133 \\
\hline $963 \mathrm{~A}-22-2$ & 183.10 & 1.705 & -1.694 \\
\hline $963 \mathrm{~A}-22-2$ & 183.52 & 1.945 & -1.661 \\
\hline $963 \mathrm{~A}-22-3$ & 183.92 & 2.024 & -1.494 \\
\hline $963 \mathrm{~A}-22-3$ & 184.32 & 1.979 & -1.338 \\
\hline $963 \mathrm{~A}-22-3$ & 184.82 & 1.499 & -0.832 \\
\hline $963 \mathrm{~A}-22-5$ & 187.02 & 1.870 & -1.151 \\
\hline $963 A-22-5$ & 187.42 & 1.605 & -1.090 \\
\hline $963 \mathrm{~A}-22-5$ & 187.82 & 1.695 & -1.105 \\
\hline $963 \mathrm{~A}-22-6$ & 188.52 & 1.479 & -1.250 \\
\hline $963 \mathrm{~A}-22-6$ & 188.92 & 1.786 & -0.907 \\
\hline $963 \mathrm{~A}-22-7$ & 189.82 & 1.751 & -0.573 \\
\hline $963 \mathrm{~A}-22-1$ & 190.52 & 0.880 & -1.816 \\
\hline $963 \mathrm{~A}-22-1$ & 190.52 & 0.933 & -1.593 \\
\hline $963 \mathrm{~A}-22-7$ & 190.62 & 1.783 & -0.880 \\
\hline $963 \mathrm{~A}-23-1$ & 190.92 & 1.338 & -1.681 \\
\hline $963 \mathrm{~A}-23-1$ & 190.92 & 1.091 & -1.782 \\
\hline $963 \mathrm{~A}-23-1$ & 191.30 & 1.661 & -0.466 \\
\hline $963 \mathrm{~A}-23-1$ & 191.30 & 1.571 & -0.748 \\
\hline $963 \mathrm{~A}-23-1$ & 191.52 & 1.961 & -1.080 \\
\hline $963 \mathrm{~A}-23-2$ & 191.82 & 2.440 & -0.536 \\
\hline $963 \mathrm{~A}-23-2$ & 192.22 & 2.638 & -0.448 \\
\hline $963 \mathrm{~A}-23-2$ & 192.62 & 1.557 & -0.877 \\
\hline $963 \mathrm{~A}-23-2$ & 193.22 & 1.740 & -0.582 \\
\hline $963 \mathrm{~A}-23-3$ & 193.92 & 1.090 & -1.331 \\
\hline $963 \mathrm{~A}-23-3$ & 194.32 & 1.448 & -0.889 \\
\hline $963 \mathrm{~A}-23-3$ & 194.72 & 1.544 & -0.848 \\
\hline $963 \mathrm{~A}-23-4$ & 195.42 & 1.723 & -0.847 \\
\hline $963 \mathrm{~B}-23-1$ & 195.64 & 0.857 & -0.966 \\
\hline $963 \mathrm{~B}-23-2$ & 196.39 & 0.326 & -2.196 \\
\hline $963 \mathrm{~B}-23-2$ & 196.73 & 1.042 & -0.592 \\
\hline $963 \mathrm{~B}-23-2$ & 197.14 & 1.644 & -0.774 \\
\hline $963 \mathrm{~A}-24-1$ & 197.62 & 1.546 & -1.670 \\
\hline $963 \mathrm{~A}-24-2$ & 197.92 & 2.181 & -1.038 \\
\hline $963 \mathrm{~A}-24-2$ & 199.12 & 1.984 & -0.647 \\
\hline $963 \mathrm{~A}-24-3$ & 199.62 & 1.263 & -0.529 \\
\hline $963 \mathrm{~A}-24-3$ & 200.02 & 1.074 & -1.186 \\
\hline $963 \mathrm{~A}-24-3$ & 200.78 & 1.017 & -1.361 \\
\hline $963 \mathrm{~A}-24-4$ & 201.12 & 1.126 & -1.180 \\
\hline
\end{tabular}




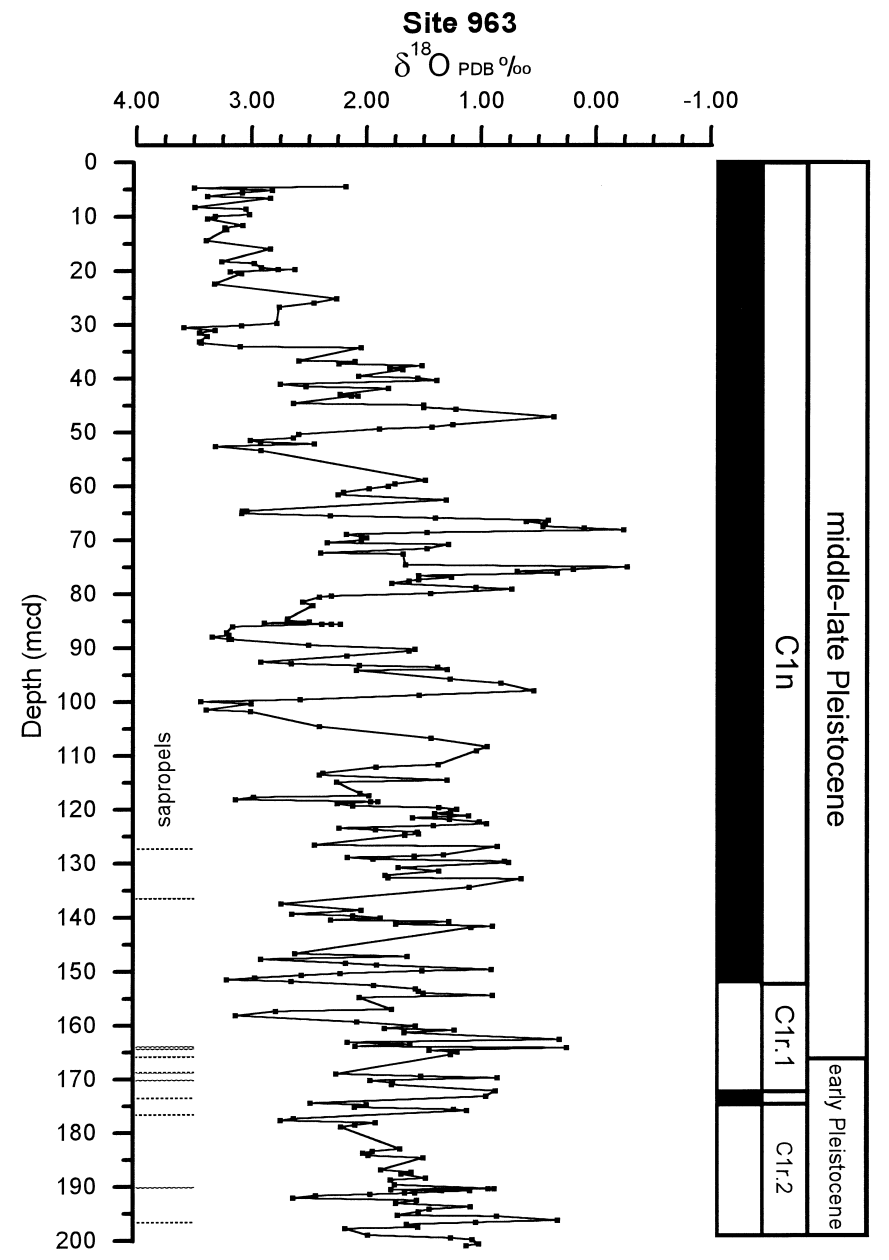

Figure 2. Site 963 oxygen isotope record of G. bulloides plotted against composite depth (mcd). The stratigraphic positions of the paleomagnetic reversals are indicated on the right. The lines on the left mark the stratigraphic positions of the sapropels.

event that began at $\sim 2.6 \mathrm{Ma}$ in the Site $964 \delta^{18} \mathrm{O}$ record was most likely a response to the same processes responsible for the formation of ice-rafted debris in the North Atlantic at $2.5 \mathrm{Ma}$ as described by Backman (1979), Ruddiman et al. (1987) and Shackleton et al. (1984).

Between 2.6 and $1.5 \mathrm{Ma}$, average $\delta^{18} \mathrm{O}$ values increase over the previous interval by $0.15 \%$ (Table 6). At $0.95 \mathrm{Ma}$, a $1.75 \%$ increase in $\delta^{18} \mathrm{O}$ occurs that most likely reflects the further intensification of Northern Hemisphere glaciation (Fig. 5). Another major increase in the oxygen isotope record at this site occurs at $0.45 \mathrm{Ma}$, and is the largest glacial-interglacial shift occurring in this part of the record (Table 6). As at Site 963 , this increase in glacial $\delta^{18} \mathrm{O}$ values marks the response to the global predominance of the 100-k.y. eccentricity cycle at this time (Ruddiman and Raymo, 1988), which resulted in colder temperatures during glacial periods. In addition, the relatively high amplitude of the $\delta^{18} \mathrm{O}$ signal reflects the presence of sapropels and represents changes in surface-water salinities in addition to changes in the global continental ice volume and sea-surface temperatures.

\section{CARBON ISOTOPES}

The present-day Mediterranean does not exhibit a large surface to bottom-water gradient in the distribution of $\delta^{13} \mathrm{C}$, and the overall en-

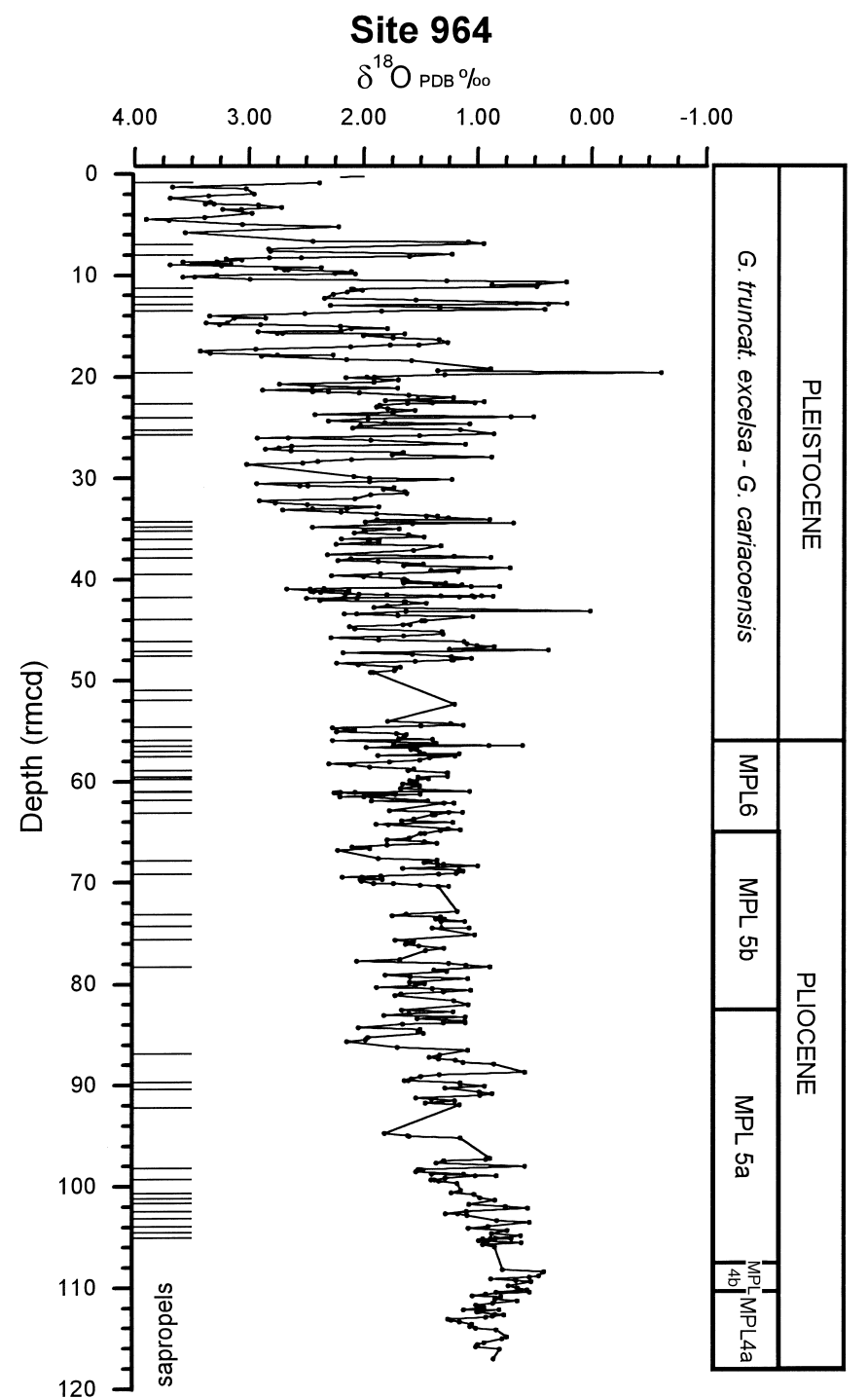

Figure 3. Site 964 oxygen isotope record of G. bulloides plotted against revised composite depth (rmcd). The lines on the left mark the stratigraphic positions of the sapropels. The planktonic foraminiferal zonations are after Cita (1975).

richment in $\delta^{13} \mathrm{C}$ is on the order of $0.5 \%$ (Duplessy, 1972; Pierre et al., 1986). This has been attributed to a combination of relatively short residence time (on the order of 100 years, Lacombe et al., 1981) and low surface-water productivity (Thunell et al., 1987; VergnaudGrazzini et al., 1990). This appears to be a relatively recent feature. According to Thunell et al. (1987), the surface to bottom-water $\delta^{13} \mathrm{C}$ gradient was as great as $3.0 \%$ o during the early Pliocene, decreasing to $\sim 1.5 \%$ o during the late Pliocene and gradually into the Pleistocene until modern-day values are reached. They concluded that the larger surface to bottom-water $\delta^{13} \mathrm{C}$ gradient reflected an estuarine circulation pattern with Atlantic water entering at depth, as opposed to the present anti-estuarine flow.

Vergnaud-Grazzini et al. (1986) reported a mean $\delta^{13} \mathrm{C}$ range of $-0.6 \%$ o to $-0.7 \%$ o for $G$. bulloides in the present-day Mediterranean on the basis of analyses of plankton tow samples. They observed that $G$. bulloides does not precipitate its calcite in full equilibrium with the ambient $\Sigma \mathrm{CO}_{2}$ and that its $\delta^{13} \mathrm{C}$ reflects the regeneration of nutrients at shallow depths (Vergnaud-Grazzini et al., 1990). Thunell (1978) classified this species as a member of the cool- 


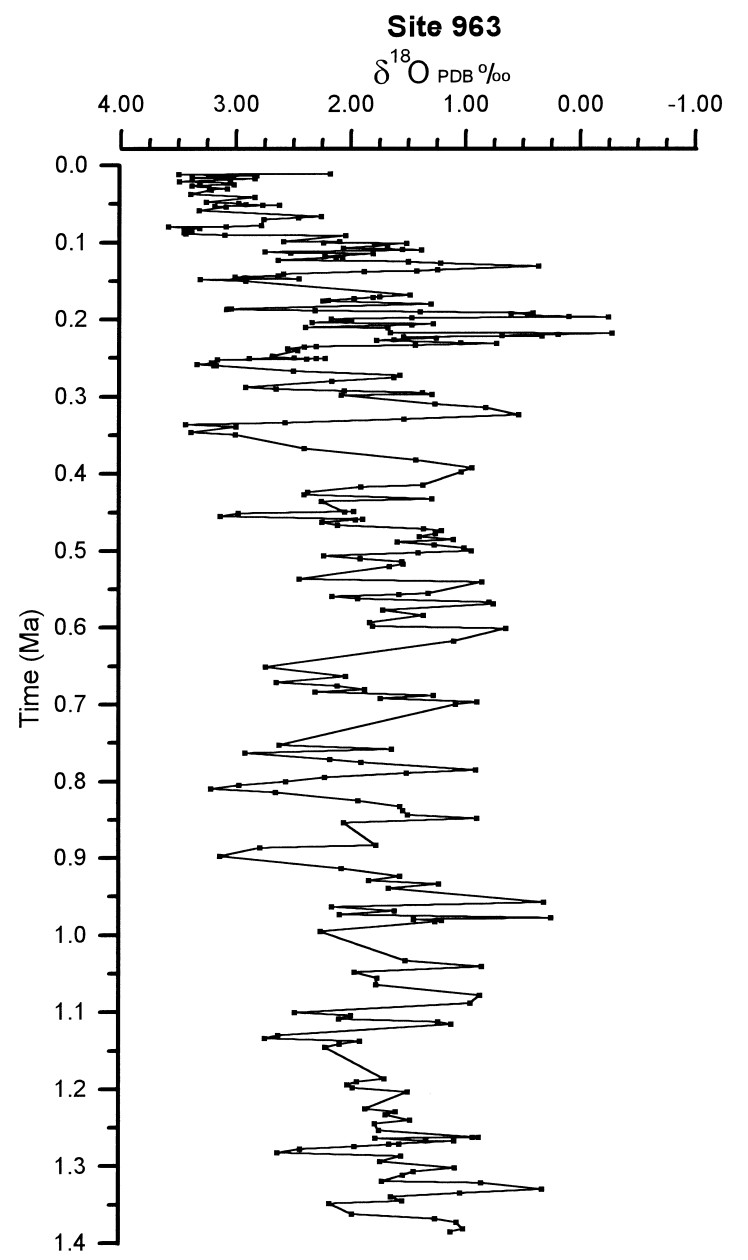

Figure 4. Site 963 oxygen isotope record of G. bulloides plotted vs. time.

Table 4. Site 963 oxygen isotope averages ( $G$, bulloides).

\begin{tabular}{lcc}
\hline $\begin{array}{c}\text { Time interval } \\
(\mathrm{Ma})\end{array}$ & $\begin{array}{c}\text { Mean } \delta^{18} \mathrm{O} \\
(\% \circ)\end{array}$ & $\begin{array}{c}\delta^{18} \mathrm{O} \text { avg. } \\
\text { deviation (\%o) }\end{array}$ \\
\hline Holocene-0.46 & 2.23 & 0.75 \\
$0.46-0.98$ & 1.82 & 0.54 \\
$0.98-1.25$ & 1.60 & 0.43 \\
\hline
\end{tabular}

subtropical assemblage in the Mediterranean, and it has been associated with upwelling environments in other localities (Thiede, 1978; Thunell and Reynolds, 1984). In their analyses of G. bulloides from Hole 653A and Site 654 in the Tyrrhenian Sea, Vergnaud-Grazzini et al. (1990) concluded that relative fluctuations in the $\delta^{13} \mathrm{C}$ of this species could be utilized for estimating the magnitude of changes in surface-water stratification. Low $\delta^{13} \mathrm{C}$ values reflect episodes of strong stratification in which nutrient-rich and isotopically light water can be regenerated at relatively shallow depths. The $\delta^{13} \mathrm{C}$ values of $G$. bulloides may also reflect changes in the $\delta^{13} \mathrm{C}$ of the $\Sigma \mathrm{CO}_{2}$ in the surface waters resulting from the introduction and remineralization of terrestrial organic matter during episodes of freshwater input into the Mediterranean (Howell et al., 1990; Thunell et al., 1990). No attempt has been made to reconstruct major circulation patterns at Site 963 from the G. bulloides $\delta^{13} \mathrm{C}$ data. Additional studies will be required to understand the impact of the surface-water changes on the formation of the MIW and its implication for sapropel formation.
Table 5. Site 964 stable isotope data (G. bulloides).

\begin{tabular}{|c|c|c|c|}
\hline $\begin{array}{l}\text { Hole, core, } \\
\text { section }\end{array}$ & $\begin{array}{l}\text { Depth } \\
\text { (rmcd) }\end{array}$ & $\begin{array}{c}\delta^{18} \mathrm{O} \\
(\% \circ, \mathrm{PDB})\end{array}$ & $\begin{array}{c}\delta^{13} \mathrm{C} \\
(\%, \text { PDB })\end{array}$ \\
\hline $964 \mathrm{~A}-1 \mathrm{H}-1$ & 0.90 & 2.39 & -1.02 \\
\hline $964 \mathrm{~A}-1 \mathrm{H}-1$ & 1.30 & 3.67 & -0.38 \\
\hline $964 \mathrm{~A}-1 \mathrm{H}-1$ & 1.48 & 3.03 & -0.35 \\
\hline $964 \mathrm{~A}-1 \mathrm{H}-2$ & 2.00 & 2.96 & 0.31 \\
\hline $964 \mathrm{~A}-1 \mathrm{H}-2$ & 2.20 & 3.36 & 0.33 \\
\hline $964 \mathrm{~A}-1 \mathrm{H}-2$ & 2.40 & 3.69 & -0.02 \\
\hline $964 \mathrm{~A}-1 \mathrm{H}-2$ & 2.80 & 3.34 & -0.55 \\
\hline $964 \mathrm{~A}-1 \mathrm{H}-2$ & 2.98 & 3.31 & -0.21 \\
\hline $964 \mathrm{~A}-1 \mathrm{H}-2$ & 2.98 & 3.39 & -0.31 \\
\hline $964 \mathrm{~A}-1 \mathrm{H}-3$ & 3.10 & 2.92 & -0.52 \\
\hline $964 \mathrm{~A}-1 \mathrm{H}-3$ & 3.30 & 2.72 & -0.59 \\
\hline $964 \mathrm{~A}-1 \mathrm{H}-3$ & 3.50 & 3.07 & -0.24 \\
\hline $964 \mathrm{~A}-1 \mathrm{H}-3$ & 3.50 & 3.23 & -0.04 \\
\hline $964 \mathrm{~A}-1 \mathrm{H}-3$ & 3.90 & 2.98 & -0.49 \\
\hline $964 \mathrm{~A}-1 \mathrm{H}-3$ & 4.30 & 3.39 & 0.04 \\
\hline $964 \mathrm{~A}-1 \mathrm{H}-3$ & 4.48 & 3.90 & 0.05 \\
\hline $964 \mathrm{~A}-1 \mathrm{H}-4$ & 4.60 & 3.70 & -0.37 \\
\hline $964 \mathrm{~A}-1 \mathrm{H}-4$ & 5.00 & 3.06 & -0.20 \\
\hline $964 \mathrm{~A}-1 \mathrm{H}-4$ & 5.20 & 2.22 & -0.61 \\
\hline $964 \mathrm{~A}-1 \mathrm{H}-4$ & 5.80 & 3.56 & -0.11 \\
\hline $964 \mathrm{~B}-1 \mathrm{H}-5$ & 6.68 & 2.44 & -0.85 \\
\hline $964 \mathrm{~B}-1 \mathrm{H}-5$ & 6.74 & 1.08 & -0.87 \\
\hline $964 \mathrm{~B}-1 \mathrm{H}-5$ & 6.88 & 0.94 & -1.22 \\
\hline $964 \mathrm{D}-2 \mathrm{H}-1$ & 7.42 & 2.83 & 0.10 \\
\hline $964 \mathrm{D}-2 \mathrm{H}-1$ & 7.66 & 2.81 & -0.92 \\
\hline $964 \mathrm{D}-2 \mathrm{H}-1$ & 7.91 & 1.22 & -1.02 \\
\hline $964 \mathrm{D}-2 \mathrm{H}-1$ & 8.14 & 1.60 & -0.59 \\
\hline $964 \mathrm{~A}-2 \mathrm{H}-1$ & 8.30 & 2.54 & -0.74 \\
\hline $964 \mathrm{~A}-2 \mathrm{H}-1$ & 8.30 & 2.83 & -0.73 \\
\hline $964 \mathrm{D}-2 \mathrm{H}-1$ & 8.37 & 3.20 & -0.86 \\
\hline $964 \mathrm{~A}-2 \mathrm{H}-2$ & 8.50 & 3.07 & -0.24 \\
\hline 964D-2H-1 & 8.69 & 3.28 & -0.71 \\
\hline $964 \mathrm{~A}-2 \mathrm{H}-2$ & 8.70 & 3.58 & -0.14 \\
\hline $964 \mathrm{~A}-2 \mathrm{H}-2$ & 8.90 & 3.16 & -0.12 \\
\hline 964D-2H-1 & 8.95 & 3.69 & -0.41 \\
\hline $964 \mathrm{~A}-2 \mathrm{H}-2$ & 9.10 & 3.24 & -0.82 \\
\hline $964 \mathrm{D}-2 \mathrm{H}-2$ & 9.28 & 2.37 & -0.78 \\
\hline $964 \mathrm{~A}-2 \mathrm{H}-2$ & 9.30 & 2.77 & -0.38 \\
\hline $964 \mathrm{~A}-2 \mathrm{H}-2$ & 9.50 & 2.69 & -0.81 \\
\hline $964 \mathrm{D}-2 \mathrm{H}-2$ & 9.50 & 2.66 & -0.79 \\
\hline $964 \mathrm{D}-2 \mathrm{H}-2$ & 9.65 & 2.11 & -0.72 \\
\hline $964 \mathrm{D}-2 \mathrm{H}-2$ & 9.81 & 2.25 & -1.21 \\
\hline $964 \mathrm{~A}-2 \mathrm{H}-2$ & 9.88 & 2.07 & -0.21 \\
\hline $964 \mathrm{D}-2 \mathrm{H}-2$ & 10.00 & 3.28 & -0.82 \\
\hline $964 \mathrm{D}-2 \mathrm{H}-2$ & 10.19 & 3.58 & -0.55 \\
\hline $964 \mathrm{~A}-2 \mathrm{H}-3$ & 10.20 & 3.48 & -0.58 \\
\hline $964 \mathrm{~A}-2 \mathrm{H}-3$ & 10.40 & 2.99 & -0.10 \\
\hline $964 \mathrm{~A}-2 \mathrm{H}-3$ & 10.60 & 1.27 & -1.05 \\
\hline $964 \mathrm{~A}-2 \mathrm{H}-3$ & 10.70 & 0.22 & -1.04 \\
\hline $964 \mathrm{~A}-2 \mathrm{H}-3$ & 10.90 & 0.47 & -1.17 \\
\hline $964 \mathrm{~A}-2 \mathrm{H}-3$ & 11.00 & 0.87 & -0.87 \\
\hline $964 \mathrm{~A}-2 \mathrm{H}-3$ & 11.16 & 0.48 & -1.06 \\
\hline $964 \mathrm{~A}-2 \mathrm{H}-3$ & 11.38 & 2.11 & -0.78 \\
\hline $964 \mathrm{~A}-2 \mathrm{H}-3$ & 11.38 & 2.09 & -0.81 \\
\hline $964 \mathrm{~A}-2 \mathrm{H}-4$ & 11.50 & 2.01 & -0.16 \\
\hline $964 \mathrm{~A}-2 \mathrm{H}-4$ & 11.70 & 2.14 & 0.26 \\
\hline $964 \mathrm{~A}-2 \mathrm{H}-4$ & 11.90 & 2.26 & 0.22 \\
\hline $964 \mathrm{~A}-2 \mathrm{H}-4$ & 12.30 & 2.34 & 0.26 \\
\hline $964 \mathrm{~A}-2 \mathrm{H}-4$ & 12.50 & 1.54 & 0.07 \\
\hline $964 \mathrm{~A}-2 \mathrm{H}-4$ & 12.80 & 0.21 & -1.07 \\
\hline $964 \mathrm{~A}-2 \mathrm{H}-4$ & 12.80 & 0.66 & -1.04 \\
\hline $964 \mathrm{~A}-2 \mathrm{H}-4$ & 12.88 & 0.38 & -1.00 \\
\hline $964 \mathrm{~A}-2 \mathrm{H}-5$ & 13.00 & 2.29 & -0.45 \\
\hline $964 \mathrm{~A}-2 \mathrm{H}-5$ & 13.22 & 1.33 & -0.74 \\
\hline $964 \mathrm{~A}-2 \mathrm{H}-5$ & 13.40 & 0.41 & -1.52 \\
\hline $964 \mathrm{~A}-2 \mathrm{H}-5$ & 13.60 & 1.84 & -0.52 \\
\hline $964 \mathrm{~A}-2 \mathrm{H}-5$ & 13.80 & 2.52 & -1.29 \\
\hline $964 \mathrm{~A}-2 \mathrm{H}-5$ & 14.00 & 3.34 & -0.42 \\
\hline $964 \mathrm{~A}-2 \mathrm{H}-5$ & 14.24 & 2.85 & -1.00 \\
\hline $964 \mathrm{~A}-2 \mathrm{H}-5$ & 14.24 & 3.13 & -0.43 \\
\hline $964 \mathrm{~A}-2 \mathrm{H}-6$ & 14.70 & 3.19 & -0.02 \\
\hline $964 \mathrm{~A}-2 \mathrm{H}-6$ & 14.70 & 3.38 & -0.89 \\
\hline $964 \mathrm{~A}-2 \mathrm{H}-6$ & 14.90 & 3.26 & -0.48 \\
\hline $964 \mathrm{~A}-2 \mathrm{H}-6$ & 14.90 & 2.90 & -0.27 \\
\hline $964 \mathrm{~A}-2 \mathrm{H}-6$ & 15.10 & 2.20 & -0.25 \\
\hline $964 \mathrm{~A}-2 \mathrm{H}-6$ & 15.30 & 1.79 & -0.10 \\
\hline $964 \mathrm{~A}-2 \mathrm{H}-6$ & 15.30 & 2.10 & -0.07 \\
\hline $964 \mathrm{~A}-2 \mathrm{H}-6$ & 15.50 & 2.20 & -0.60 \\
\hline $964 \mathrm{~B}-2 \mathrm{H}-4$ & 15.61 & 2.92 & -0.27 \\
\hline $964 \mathrm{~A}-2 \mathrm{H}-6$ & 15.74 & 2.75 & -0.69 \\
\hline $964 \mathrm{~A}-2 \mathrm{H}-6$ & 15.74 & 2.71 & -0.51 \\
\hline $964 \mathrm{~B}-2 \mathrm{H}-4$ & 15.83 & 1.64 & -1.01 \\
\hline $964 \mathrm{~B}-2 \mathrm{H}-4$ & 16.01 & 2.00 & -0.39 \\
\hline 964B-2H-4 & 16.20 & 1.74 & -0.44 \\
\hline $964 \mathrm{~B}-2 \mathrm{H}-4$ & 16.38 & 1.34 & 0.25 \\
\hline 964B-2H-5 & 16.70 & 1.26 & -0.49 \\
\hline 964B-2H-5 & 16.90 & 1.51 & -1.00 \\
\hline $964 \mathrm{~B}-2 \mathrm{H}-5$ & 16.90 & 1.76 & -0.95 \\
\hline 964B-2H-5 & 17.10 & 2.11 & -0.09 \\
\hline
\end{tabular}


Table 5 (continued).

\begin{tabular}{|c|c|c|c|}
\hline $\begin{array}{c}\text { Hole, core, } \\
\text { section }\end{array}$ & $\begin{array}{l}\text { Depth } \\
\text { (rmcd) }\end{array}$ & $\begin{array}{c}\delta^{18} \mathrm{O} \\
(\%, \mathrm{PDB})\end{array}$ & $\begin{array}{c}\delta^{13} \mathrm{C} \\
(\% o, \mathrm{PDB})\end{array}$ \\
\hline 964B-2H-5 & 17.30 & 2.94 & -0.84 \\
\hline $964 \mathrm{~B}-2 \mathrm{H}-5$ & 17.50 & 3.43 & -0.12 \\
\hline 964B-2H-5 & 17.70 & 3.34 & -0.61 \\
\hline $964 \mathrm{~B}-2 \mathrm{H}-5$ & 17.88 & 2.75 & -0.64 \\
\hline $964 \mathrm{~B}-2 \mathrm{H}-6$ & 17.90 & 2.26 & -1.06 \\
\hline $964 \mathrm{~A}-3 \mathrm{H}-1$ & 17.97 & 2.89 & -0.69 \\
\hline $964 \mathrm{~A}-3 \mathrm{H}-1$ & 18.38 & 2.15 & -0.35 \\
\hline $964 \mathrm{~A}-3 \mathrm{H}-1$ & 18.45 & 1.58 & -0.12 \\
\hline 964A-3H-2 & 19.25 & 0.88 & -0.82 \\
\hline $964 \mathrm{~A}-3 \mathrm{H}-2$ & 19.45 & 1.35 & -0.26 \\
\hline $964 \mathrm{~A}-3 \mathrm{H}-2$ & 19.66 & -0.61 & -1.84 \\
\hline $964 \mathrm{~A}-3 \mathrm{H}-2$ & 19.84 & 1.29 & -0.69 \\
\hline $964 \mathrm{~A}-3 \mathrm{H}-2$ & 20.04 & 1.97 & -0.27 \\
\hline $964 \mathrm{~A}-3 \mathrm{H}-2$ & 20.04 & 1.90 & -0.61 \\
\hline $964 \mathrm{~A}-3 \mathrm{H}-3$ & 20.16 & 2.15 & -0.74 \\
\hline $964 \mathrm{~A}-3 \mathrm{H}-3$ & 20.36 & 1.69 & -0.54 \\
\hline $964 \mathrm{~A}-3 \mathrm{H}-3$ & 20.56 & 1.91 & -0.65 \\
\hline $964 \mathrm{~A}-3 \mathrm{H}-3$ & 20.76 & 2.73 & -0.34 \\
\hline $964 \mathrm{~A}-3 \mathrm{H}-3$ & 20.96 & 2.45 & -0.43 \\
\hline $964 \mathrm{~A}-3 \mathrm{H}-3$ & 21.16 & 1.70 & -0.80 \\
\hline $964 \mathrm{~A}-3 \mathrm{H}-3$ & 21.36 & 2.88 & 0.27 \\
\hline $964 \mathrm{~A}-3 \mathrm{H}-3$ & 21.54 & 2.31 & 0.19 \\
\hline $964 \mathrm{~A}-3 \mathrm{H}-3$ & 21.54 & 2.44 & 0.19 \\
\hline $964 \mathrm{~A}-3 \mathrm{H}-4$ & 21.66 & 2.03 & 0.02 \\
\hline $964 \mathrm{~A}-3 \mathrm{H}-4$ & 21.86 & 1.60 & -0.09 \\
\hline $964 \mathrm{~A}-3 \mathrm{H}-4$ & 22.06 & 1.21 & -0.44 \\
\hline $964 \mathrm{~A}-3 \mathrm{H}-4$ & 22.26 & 1.52 & -0.40 \\
\hline $964 \mathrm{~A}-3 \mathrm{H}-5$ & 22.36 & 1.81 & -0.35 \\
\hline $964 \mathrm{~A}-3 \mathrm{H}-4$ & 22.46 & 0.94 & -1.58 \\
\hline $964 \mathrm{~A}-3 \mathrm{H}-4$ & 22.58 & $\begin{array}{l}1.39 \\
\end{array}$ & $\begin{array}{l}-1.32 \\
-1.32\end{array}$ \\
\hline $964 \mathrm{~A}-3 \mathrm{H}-4$ & 22.58 & 1.02 & -1.51 \\
\hline $964 \mathrm{~A}-3 \mathrm{H}-4$ & 22.66 & 1.61 & -0.77 \\
\hline $964 \mathrm{~A}-3 \mathrm{H}-4$ & 22.86 & 1.86 & -0.20 \\
\hline $964 \mathrm{~A}-3 \mathrm{H}-4$ & 23.04 & 1.88 & -0.52 \\
\hline $964 \mathrm{~A}-3 \mathrm{H}-5$ & 23.16 & 1.79 & -0.74 \\
\hline $964 \mathrm{~A}-3 \mathrm{H}-5$ & 23.36 & 1.54 & -0.61 \\
\hline $964 \mathrm{~A}-3 \mathrm{H}-5$ & 23.56 & 1.74 & -1.00 \\
\hline $964 \mathrm{~A}-3 \mathrm{H}-5$ & 23.76 & 2.42 & -1.07 \\
\hline $964 \mathrm{~A}-3 \mathrm{H}-5$ & 23.98 & 0.70 & -1.45 \\
\hline $964 \mathrm{~A}-3 \mathrm{H}-5$ & 23.98 & 0.50 & -1.73 \\
\hline $964 \mathrm{~A}-3 \mathrm{H}-5$ & 24.16 & 1.96 & -1.12 \\
\hline $964 \mathrm{~A}-3 \mathrm{H}-5$ & 24.36 & 2.30 & -0.72 \\
\hline $964 \mathrm{~A}-3 \mathrm{H}-5$ & 24.54 & 1.81 & -0.04 \\
\hline $964 \mathrm{~A}-3 \mathrm{H}-6$ & 24.66 & 1.06 & -0.35 \\
\hline $964 \mathrm{~A}-3 \mathrm{H}-6$ & 24.86 & 2.03 & -0.71 \\
\hline $964 \mathrm{~A}-3 \mathrm{H}-6$ & 25.06 & 2.09 & -0.75 \\
\hline $964 \mathrm{~A}-3 \mathrm{H}-6$ & 25.26 & 1.15 & -1.48 \\
\hline 964B-3H-5 & 25.64 & 0.85 & -1.55 \\
\hline 964B-3H-5 & 25.85 & 1.51 & -0.47 \\
\hline $964 \mathrm{~B}-3 \mathrm{H}-5$ & 26.06 & 2.93 & -0.73 \\
\hline 964B-3H-5 & 26.06 & 2.66 & -0.78 \\
\hline $964 \mathrm{~B}-3 \mathrm{H}-5$ & 26.26 & 1.93 & -1.25 \\
\hline $964 \mathrm{~B}-3 \mathrm{H}-5$ & 26.69 & 1.10 & -1.57 \\
\hline $964 \mathrm{~B}-3 \mathrm{H}-5$ & 26.87 & 2.62 & -1.15 \\
\hline $964 \mathrm{~B}-3 \mathrm{H}-5$ & 27.03 & 2.74 & -0.83 \\
\hline $964 \mathrm{~B}-3 \mathrm{H}-6$ & 27.13 & 2.86 & -0.70 \\
\hline 964B-3H-6 & 27.31 & 2.63 & -0.82 \\
\hline $964 \mathrm{~B}-3 \mathrm{H}-6$ & 27.48 & 1.65 & -0.90 \\
\hline $964 \mathrm{~B}-3 \mathrm{H}-6$ & 27.74 & 1.74 & -0.67 \\
\hline $964 \mathrm{~B}-3 \mathrm{H}-6$ & 27.96 & 0.87 & -1.44 \\
\hline 964B-3H-6 & 28.16 & 2.10 & 0.01 \\
\hline $964 \mathrm{~B}-3 \mathrm{H}-6$ & 28.36 & 2.40 & -0.71 \\
\hline 964B-3H-6 & 28.56 & 2.53 & -0.28 \\
\hline 964B-3H-7 & 28.64 & 3.02 & -0.35 \\
\hline $964 \mathrm{D}-4 \mathrm{H}-1$ & 29.84 & 2.08 & -0.58 \\
\hline 964D-4H-1 & 30.02 & 1.94 & -0.10 \\
\hline 964D-4H-2 & 30.14 & 1.22 & -0.50 \\
\hline $964 \mathrm{D}-4 \mathrm{H}-2$ & 30.38 & 1.95 & -0.83 \\
\hline 964D-4H-2 & 30.54 & 2.93 & -0.70 \\
\hline $964 \mathrm{D}-4 \mathrm{H}-2$ & 30.76 & 2.56 & -0.60 \\
\hline 964D-4H-2 & 30.76 & 2.48 & -0.70 \\
\hline $964 \mathrm{D}-4 \mathrm{H}-2$ & 30.94 & 1.73 & -0.44 \\
\hline 964D-4H-2 & 31.14 & 1.82 & -0.86 \\
\hline 964D-4H-2 & 31.34 & 1.63 & -1.08 \\
\hline $964 \mathrm{D}-4 \mathrm{H}-2$ & 31.52 & 1.62 & -0.83 \\
\hline $964 \mathrm{D}-4 \mathrm{H}-3$ & 31.64 & 1.93 & -0.44 \\
\hline 964D-4H-3 & 32.04 & 2.07 & -0.75 \\
\hline $964 \mathrm{D}-4 \mathrm{H}-3$ & 32.24 & 2.91 & -1.39 \\
\hline 964D-4H-3 & 32.44 & 2.77 & -0.98 \\
\hline 964D-4H-3 & 32.64 & 2.49 & -0.81 \\
\hline $964 \mathrm{D}-4 \mathrm{H}-3$ & 32.84 & 1.86 & -1.29 \\
\hline $964 \mathrm{D}-4 \mathrm{H}-3$ & 33.02 & 2.14 & -0.99 \\
\hline $964 \mathrm{D}-4 \mathrm{H}-3$ & 33.02 & 2.44 & -0.69 \\
\hline 964D-4H-4 & 33.14 & 2.70 & -0.70 \\
\hline $964 \mathrm{D}-4 \mathrm{H}-4$ & 33.3 & 2.20 & -0.77 \\
\hline $964 \mathrm{D}-4 \mathrm{H}-4$ & 33.54 & 1.88 & -0.79 \\
\hline 964D-4H-4 & 33.76 & 1.35 & -1.25 \\
\hline & & & \\
\hline
\end{tabular}

\begin{tabular}{|c|c|c|c|}
\hline $\begin{array}{l}\text { Hole, core, } \\
\text { section }\end{array}$ & $\begin{array}{l}\text { Depth } \\
\text { (rmcd) }\end{array}$ & $\begin{array}{c}\delta^{18} \mathrm{O} \\
(\%, \mathrm{PDB})\end{array}$ & $\begin{array}{c}\delta^{13} \mathrm{C} \\
(\% o, \text { PDB })\end{array}$ \\
\hline $964 \mathrm{D}-4 \mathrm{H}-4$ & 33.94 & 1.25 & -0.69 \\
\hline 964D-4H-4 & 34.14 & 0.89 & -1.08 \\
\hline 964D-4H-4 & 34.14 & 1.88 & -0.74 \\
\hline 964D-4H-4 & 34.36 & 1.98 & -0.11 \\
\hline $964 \mathrm{D}-4 \mathrm{H}-4$ & 34.48 & 0.68 & -2.04 \\
\hline 964D-4H-4 & 34.52 & 1.56 & -0.99 \\
\hline $964 \mathrm{D}-4 \mathrm{H}-5$ & 34.84 & 2.44 & $\begin{array}{l}-0.07 \\
-0.07\end{array}$ \\
\hline $964 \mathrm{D}-4 \mathrm{H}-5$ & 35.04 & 1.68 & -0.75 \\
\hline $964 \mathrm{D}-4 \mathrm{H}-5$ & 35.26 & 1.99 & -0.50 \\
\hline $964 \mathrm{D}-4 \mathrm{H}-5$ & 35.44 & 2.08 & -0.67 \\
\hline $964 \mathrm{D}-4 \mathrm{H}-5$ & 35.64 & 1.60 & -0.63 \\
\hline 964D-4H-5 & 35.84 & 1.47 & -1.11 \\
\hline 964D-4H-5 & 36.02 & $\begin{array}{l}1.47 \\
2.19\end{array}$ & $\begin{array}{l}-1.11 \\
-0.82\end{array}$ \\
\hline $964 \mathrm{D}-4 \mathrm{H}-6$ & 36.08 & 1.94 & -0.97 \\
\hline $964 \mathrm{D}-4 \mathrm{H}-6$ & 36.14 & 1.86 & -1.03 \\
\hline $964 \mathrm{D}-4 \mathrm{H}-6$ & 36.34 & 1.86 & -1.08 \\
\hline 964D-4H-6 & 36.34 & 1.95 & -0.97 \\
\hline $964 \mathrm{D}-4 \mathrm{H}-6$ & 36.54 & 2.23 & -0.64 \\
\hline $964 \mathrm{D}-4 \mathrm{H}-6$ & 36.72 & 1.32 & $\begin{array}{l}-0.04 \\
-0.85\end{array}$ \\
\hline $964 \mathrm{~B}-4 \mathrm{H}-3$ & 37.16 & 1.56 & -0.66 \\
\hline $964 \mathrm{~B}-4 \mathrm{H}-3$ & 37.56 & 2.31 & -0.51 \\
\hline $964 \mathrm{~B}-4 \mathrm{H}-3$ & 37.76 & 1.20 & -0.76 \\
\hline $964 \mathrm{~B}-4 \mathrm{H}-3$ & 37.84 & 0.88 & -1.87 \\
\hline $964 \mathrm{~B}-4 \mathrm{H}-3$ & 37.96 & $\begin{array}{l}2.00 \\
2.10\end{array}$ & $\begin{array}{l}-1.01 \\
-1.33\end{array}$ \\
\hline $964 \mathrm{~B}-4 \mathrm{H}-3$ & 38.14 & 2.22 & -1.16 \\
\hline $964 \mathrm{~B}-4 \mathrm{H}-4$ & 38.26 & 1.87 & -0.68 \\
\hline $964 \mathrm{~B}-4 \mathrm{H}-4$ & 38.46 & $\begin{array}{l}1.47 \\
1.47\end{array}$ & -0.69 \\
\hline $964 \mathrm{~B}-4 \mathrm{H}-4$ & 38.66 & 1.64 & -0.45 \\
\hline 964B-4H-4 & 38.86 & 0.71 & -1.04 \\
\hline $964 \mathrm{~B}-4 \mathrm{H}-4$ & 39.12 & 1.40 & $\begin{array}{l}-1.04 \\
-0.90\end{array}$ \\
\hline $964 \mathrm{~B}-4 \mathrm{H}-4$ & 39.26 & $\begin{array}{l}1.17 \\
1.17\end{array}$ & -0.77 \\
\hline 964B-4H-4 & 39.46 & 1.85 & -1.08 \\
\hline $964 \mathrm{~B}-4 \mathrm{H}-4$ & 39.64 & 2.27 & $\begin{array}{l}-0.80 \\
-0.80\end{array}$ \\
\hline $964 \mathrm{~B}-4 \mathrm{H}-5$ & 39.76 & 2.00 & -0.99 \\
\hline $964 \mathrm{~B}-4 \mathrm{H}-5$ & 40.00 & 1.64 & -0.95 \\
\hline $964 \mathrm{~B}-4 \mathrm{H}-5$ & 40.16 & $\begin{array}{l}1.64 \\
1.61\end{array}$ & -0.71 \\
\hline $964 \mathrm{~B}-4 \mathrm{H}-5$ & 40.16 & 1.65 & -0.53 \\
\hline $964 \mathrm{~B}-4 \mathrm{H}-5$ & 40.36 & 1.28 & -0.46 \\
\hline $964 \mathrm{~B}-4 \mathrm{H}-5$ & 40.36 & 1.64 & -0.53 \\
\hline $964 \mathrm{~B}-4 \mathrm{H}-5$ & 40.56 & 1.13 & -0.94 \\
\hline $964 \mathrm{~B}-4 \mathrm{H}-5$ & 40.56 & 1.37 & -0.58 \\
\hline $964 \mathrm{~A}-5-1$ & 40.71 & 1.05 & -1.02 \\
\hline $964 \mathrm{~A}-5-1$ & 40.71 & 0.80 & $\begin{array}{l}-1.02 \\
-0.78\end{array}$ \\
\hline $964 \mathrm{~A}-5-1$ & 40.88 & 2.34 & -0.93 \\
\hline 964B-4H-5 & 40.98 & 2.46 & -0.74 \\
\hline $964 \mathrm{~B}-4 \mathrm{H}-5$ & 40.98 & 2.67 & -0.74 \\
\hline $964 \mathrm{~A}-5 \mathrm{H}-1$ & 41.10 & 2.45 & -0.77 \\
\hline $964 \mathrm{~B}-4 \mathrm{H}-5$ & 41.14 & 2.27 & -1.19 \\
\hline $964 \mathrm{~B}-4 \mathrm{H}-5$ & $\begin{array}{l}41.14 \\
41.14\end{array}$ & 2.12 & $\begin{array}{l}-1.19 \\
-1.26\end{array}$ \\
\hline $964 \mathrm{~B}-4 \mathrm{H}-6$ & 41.20 & 2.46 & -0.22 \\
\hline $964 \mathrm{~B}-4 \mathrm{H}-6$ & 41.26 & 2.43 & -0.44 \\
\hline $964 \mathrm{~A}-5 \mathrm{H}-1$ & 41.32 & 2.37 & -0.33 \\
\hline $964 \mathrm{~B}-4 \mathrm{H}-6$ & 41.46 & 2.03 & -0.57 \\
\hline $964 \mathrm{~B}-4 \mathrm{H}-6$ & $\begin{array}{l}41.40 \\
41.46\end{array}$ & 2.15 & -0.35 \\
\hline 964A-5H-1 & $\begin{array}{l}41.40 \\
41.52\end{array}$ & 1.79 & $\begin{array}{l}-0.33 \\
-0.22\end{array}$ \\
\hline $964 \mathrm{~A}-5 \mathrm{H}-1$ & 41.64 & 1.32 & -0.42 \\
\hline 964B-4H-6 & 41.68 & 1.04 & -1.78 \\
\hline 964B-4H-6 & 41.68 & 0.96 & -1.57 \\
\hline $964 \mathrm{~A}-5 \mathrm{H}-1$ & 41.72 & 0.86 & -1.25 \\
\hline $964 \mathrm{~B}-4 \mathrm{H}-6$ & 41.74 & $\begin{array}{l}.00 \\
1.15\end{array}$ & -1.07 \\
\hline $964 \mathrm{~B}-4 \mathrm{H}-6$ & 41.74 & 1.02 & -1.49 \\
\hline $964 \mathrm{~A}-5 \mathrm{H}-1$ & 41.87 & 2.49 & -0.37 \\
\hline 964B-4H-6 & 41.88 & 2.05 & -1.04 \\
\hline $964 \mathrm{~B}-4 \mathrm{H}-6$ & 41.88 & 2.18 & -1.12 \\
\hline $964 \mathrm{~A}-5 \mathrm{H}-1$ & 42.10 & 2.38 & -0.71 \\
\hline $964 \mathrm{~A}-5 \mathrm{H}-2$ & 42.22 & 1.64 & -0.03 \\
\hline $964 \mathrm{~A}-5 \mathrm{H}-2$ & 42.22 & 1.63 & -0.46 \\
\hline $964 \mathrm{~A}-5 \mathrm{H}-2$ & 42.42 & 1.44 & -0.60 \\
\hline $964 \mathrm{~A}-5 \mathrm{H}-2$ & 42.62 & 1.78 & -0.33 \\
\hline $964 \mathrm{~A}-5 \mathrm{H}-2$ & 42.82 & 1.90 & -0.48 \\
\hline $964 \mathrm{~A}-5 \mathrm{H}-2$ & 43.18 & 0.01 & -1.80 \\
\hline $964 \mathrm{~A}-5 \mathrm{H}-2$ & 43.22 & $\begin{array}{l}0.61 \\
1.62\end{array}$ & $\begin{array}{l}-1.00 \\
-1.17\end{array}$ \\
\hline $964 \mathrm{~A}-5 \mathrm{H}-2$ & 43.42 & 2.06 & -0.59 \\
\hline $964 \mathrm{~A}-5 \mathrm{H}-2$ & 43.42 & 2.16 & -0.26 \\
\hline $964 \mathrm{~A}-5 \mathrm{H}-2$ & 43.60 & $\begin{array}{l}1.69 \\
1.69\end{array}$ & -0.27 \\
\hline $964 \mathrm{~A}-5 \mathrm{H}-3$ & 43.72 & 1.04 & -0.52 \\
\hline $964 \mathrm{~A}-5 \mathrm{H}-3$ & 44.12 & 1.45 & -0.52 \\
\hline $964 \mathrm{~A}-5 \mathrm{H}-3$ & 44.12 & 1.48 & -0.85 \\
\hline $964 \mathrm{~A}-5 \mathrm{H}-3$ & 44.52 & 1.65 & -0.66 \\
\hline $964 \mathrm{~A}-5 \mathrm{H}-3$ & 44.52 & 1.59 & -0.76 \\
\hline $964 \mathrm{~A}-5 \mathrm{H}-3$ & 44.72 & 2.12 & -0.36 \\
\hline $964 \mathrm{~A}-5 \mathrm{H}-3$ & 44.94 & 2.07 & -0.35 \\
\hline $964 \mathrm{~A}-5 \mathrm{H}-4$ & 45.22 & 1.31 & -0.42 \\
\hline 964A-5H-4 & 45.44 & $\begin{array}{l}1.31 \\
1.30\end{array}$ & $\begin{array}{l}-0.42 \\
-0.92\end{array}$ \\
\hline $964 \mathrm{~A}-5 \mathrm{H}-4$ & 45.62 & 1.64 & -1.16 \\
\hline $964 \mathrm{~A}-5 \mathrm{H}-4$ & 45.82 & 2.28 & -0.18 \\
\hline $964 \mathrm{~A}-5 \mathrm{H}-4$ & 46.02 & 1.86 & -0.21 \\
\hline & & & \\
\hline
\end{tabular}


Table 5 (continued).

\begin{tabular}{|c|c|c|c|}
\hline $\begin{array}{l}\text { Hole, core, } \\
\text { section }\end{array}$ & $\begin{array}{l}\text { Depth } \\
\text { (rmcd) }\end{array}$ & $\begin{array}{c}\delta^{18} \mathrm{O} \\
(\%, \mathrm{PDB})\end{array}$ & $\begin{array}{c}\delta^{13} \mathrm{C} \\
(\%, \text { PDB })\end{array}$ \\
\hline $964 \mathrm{~A}-5 \mathrm{H}-4$ & 46.44 & 1.09 & -0.55 \\
\hline $964 \mathrm{~A}-5 \mathrm{H}-4$ & 46.60 & 1.00 & -0.82 \\
\hline $964 \mathrm{~A}-5 \mathrm{H}-5$ & 46.72 & 0.85 & -1.10 \\
\hline $964 \mathrm{~A}-5 \mathrm{H}-5$ & 46.93 & 1.24 & 0.06 \\
\hline $964 \mathrm{~A}-5 \mathrm{H}-5$ & 47.08 & 0.37 & -1.15 \\
\hline $964 \mathrm{~A}-5 \mathrm{H}-5$ & 47.28 & 2.17 & 0.47 \\
\hline $964 \mathrm{~A}-5 \mathrm{H}-5$ & $\begin{array}{l}47.47 \\
47.20\end{array}$ & 1.57 & $\begin{array}{l}0.41 \\
-0.09\end{array}$ \\
\hline $964 \mathrm{~A}-5 \mathrm{H}-5$ & 47.66 & 1.23 & 0.06 \\
\hline $964 \mathrm{~A}-5 \mathrm{H}-5$ & 47.88 & 1.05 & -0.21 \\
\hline $964 \mathrm{~A}-5 \mathrm{H}-5$ & 48.04 & 1.22 & -0.28 \\
\hline $964 \mathrm{~A}-5 \mathrm{H}-5$ & 48.04 & 1.21 & -0.60 \\
\hline $964 \mathrm{~A}-5 \mathrm{H}-6$ & 48.14 & 1.54 & -0.79 \\
\hline $964 \mathrm{~A}-5 \mathrm{H}-6$ & $\begin{array}{l}40.14 \\
48.32\end{array}$ & $\begin{array}{l}1.24 \\
2.23\end{array}$ & -0.01 \\
\hline $964 \mathrm{~A}-5 \mathrm{H}-6$ & 48.51 & 2.04 & 0.24 \\
\hline $964 \mathrm{~A}-5 \mathrm{H}-6$ & 48.70 & 1.67 & 0.20 \\
\hline $964 \mathrm{~A}-5 \mathrm{H}-6$ & 48.89 & 1.71 & 0.16 \\
\hline $964 \mathrm{~A}-5 \mathrm{H}-6$ & 49.08 & 1.72 & -0.37 \\
\hline $964 \mathrm{~A}-5 \mathrm{H}-6$ & 49.28 & 1.93 & -0.31 \\
\hline $964 \mathrm{~A}-5 \mathrm{H}-6$ & 49.28 & 1.91 & $\begin{array}{l}-0.05 \\
-0.05\end{array}$ \\
\hline $964 \mathrm{D}-6 \mathrm{H}-1$ & 52.40 & 1.19 & -1.03 \\
\hline $964 \mathrm{D}-6 \mathrm{H}-2$ & 54.10 & 1.78 & -0.46 \\
\hline $964 \mathrm{D}-6 \mathrm{H}-2$ & 54.32 & 1.23 & -0.61 \\
\hline $964 \mathrm{D}-6 \mathrm{H}-2$ & 54.48 & 1.12 & -0.56 \\
\hline $964 \mathrm{D}-6 \mathrm{H}-2$ & 54.54 & 1.49 & -0.17 \\
\hline $964 \mathrm{D}-6 \mathrm{H}-2$ & 54.72 & 2.26 & -0.15 \\
\hline $964 \mathrm{D}-6 \mathrm{H}-2$ & 54.92 & 2.07 & -0.46 \\
\hline $964 \mathrm{D}-6 \mathrm{H}-2$ & 55.10 & 2.23 & -0.15 \\
\hline $964 \mathrm{D}-6 \mathrm{H}-2$ & 55.30 & 1.70 & -0.44 \\
\hline 964D-6H-3 & 55.42 & 1.62 & -0.46 \\
\hline $964 \mathrm{D}-6 \mathrm{H}-3$ & 55.60 & $\begin{array}{l}1.64 \\
\end{array}$ & -0.13 \\
\hline $964 \mathrm{D}-6 \mathrm{H}-3$ & 55.82 & $\begin{array}{l}1.64 \\
1.68\end{array}$ & $\begin{array}{l}-0.13 \\
-0.79\end{array}$ \\
\hline 964D-6H-3 & 55.88 & 1.39 & -1.51 \\
\hline $964 \mathrm{D}-6 \mathrm{H}-3$ & 55.98 & 2.27 & $\begin{array}{l}-0.35 \\
-0.35\end{array}$ \\
\hline $964 \mathrm{D}-6 \mathrm{H}-3$ & 56.24 & 1.36 & -1.14 \\
\hline 964D-6H-3 & 56.42 & 1.73 & -0.66 \\
\hline $964 \mathrm{D}-6 \mathrm{H}-3$ & 56.48 & 0.60 & -1.90 \\
\hline $964 \mathrm{D}-6 \mathrm{H}-3$ & 56.48 & 0.89 & $\begin{array}{l}-1.90 \\
-1.87\end{array}$ \\
\hline $964 \mathrm{D}-6 \mathrm{H}-3$ & 56.64 & 1.97 & -0.76 \\
\hline $964 \mathrm{D}-6 \mathrm{H}-3$ & 56.80 & 1.53 & -1.09 \\
\hline $964 \mathrm{D}-6 \mathrm{H}-4$ & 56.92 & 1.58 & -0.88 \\
\hline $964 \mathrm{D}-6 \mathrm{H}-4$ & 57.12 & 1.50 & -1.23 \\
\hline $964 \mathrm{D}-6 \mathrm{H}-4$ & 57.32 & 1.46 & -0.48 \\
\hline 964D-6H-4 & 57.32 & $\begin{array}{l}1.40 \\
1.15\end{array}$ & $\begin{array}{l}-0.40 \\
-0.67\end{array}$ \\
\hline $964 \mathrm{D}-6 \mathrm{H}-4$ & 57.48 & 1.87 & -0.35 \\
\hline $964 \mathrm{D}-6 \mathrm{H}-4$ & 57.50 & 1.19 & 0.15 \\
\hline $964 \mathrm{D}-6 \mathrm{H}-4$ & 57.72 & 1.41 & 0.13 \\
\hline $964 \mathrm{D}-6 \mathrm{H}-4$ & 57.92 & 1.50 & -0.04 \\
\hline $964 \mathrm{D}-6 \mathrm{H}-4$ & 58.12 & 1.77 & -1.06 \\
\hline $964 \mathrm{D}-6 \mathrm{H}-4$ & 58.30 & 2.30 & $\begin{array}{r}-1.00 \\
0.07\end{array}$ \\
\hline $964 \mathrm{D}-6 \mathrm{H}-5$ & 58.42 & 2.11 & -0.17 \\
\hline 964D-6H-5 & 58.62 & 1.94 & -0.01 \\
\hline $964 \mathrm{D}-6 \mathrm{H}-5$ & 58.80 & 1.55 & -0.41 \\
\hline $964 \mathrm{D}-6 \mathrm{H}-5$ & 58.98 & 1.60 & -0.52 \\
\hline 964D-6H-5 & 59.20 & 1.26 & -0.28 \\
\hline $964 \mathrm{D}-6 \mathrm{H}-5$ & 59.52 & 1.26 & -0.11 \\
\hline $964 \mathrm{D}-6 \mathrm{H}-5$ & 59.62 & 1.51 & -0.41 \\
\hline $964 \mathrm{D}-6 \mathrm{H}-5$ & 59.80 & 1.42 & -0.47 \\
\hline 964D-6H-6 & 59.92 & 1.59 & -0.23 \\
\hline $964 \mathrm{D}-6 \mathrm{H}-6$ & 60.12 & 1.52 & -0.19 \\
\hline $964 \mathrm{D}-6 \mathrm{H}-6$ & 60.32 & 1.65 & 0.25 \\
\hline $964 \mathrm{D}-6 \mathrm{H}-6$ & 60.48 & 1.50 & 0.17 \\
\hline $964 \mathrm{D}-6 \mathrm{H}-6$ & 60.74 & 1.67 & -0.58 \\
\hline $964 \mathrm{D}-6 \mathrm{H}-6$ & 61.02 & 1.06 & -1.42 \\
\hline $964 \mathrm{~B}-6 \mathrm{H}-5$ & 61.07 & 2.06 & -0.46 \\
\hline $964 \mathrm{~B}-6 \mathrm{H}-5$ & 61.07 & 2.19 & -0.15 \\
\hline $964 \mathrm{D}-6 \mathrm{H}-6$ & 61.14 & 2.22 & -0.18 \\
\hline $964 \mathrm{D}-6 \mathrm{H}-6$ & 61.14 & 2.25 & -0.30 \\
\hline $964 \mathrm{~B}-6 \mathrm{H}-5$ & 61.30 & 1.71 & 0.26 \\
\hline $964 \mathrm{~B}-6 \mathrm{H}-5$ & 61.30 & 1.50 & -0.48 \\
\hline $964 \mathrm{~B}-6 \mathrm{H}-5$ & 61.58 & 2.20 & 0.43 \\
\hline $964 \mathrm{~B}-6 \mathrm{H}-5$ & 61.58 & 1.99 & 0.16 \\
\hline $964 \mathrm{~B}-6 \mathrm{H}-5$ & 61.98 & 1.43 & 0.15 \\
\hline 964B-6H-5 & 61.98 & 1.92 & 0.53 \\
\hline $964 \mathrm{~B}-6 \mathrm{H}-5$ & 62.16 & 1.20 & -0.14 \\
\hline $964 \mathrm{~B}-6 \mathrm{H}-5$ & 62.16 & 1.29 & 0.17 \\
\hline $964 \mathrm{D}-7 \mathrm{H}-1$ & 62.95 & 1.76 & 0.67 \\
\hline $964 \mathrm{D}-7 \mathrm{H}-1$ & 63.14 & 1.12 & 0.01 \\
\hline 964D-7H-1 & 63.14 & 1.25 & 0.46 \\
\hline $964 \mathrm{D}-7 \mathrm{H}-1$ & 63.34 & 1.36 & 0.15 \\
\hline 964D-7H-1 & 63.34 & 1.39 & 0.29 \\
\hline 964D-7H-1 & 63.74 & 1.55 & -0.62 \\
\hline $964 \mathrm{D}-7 \mathrm{H}-1$ & 63.94 & 1.66 & -0.58 \\
\hline $964 \mathrm{D}-7 \mathrm{H}-1$ & 64.12 & 1.21 & -0.36 \\
\hline $964 \mathrm{~A}-7 \mathrm{H}-1$ & 64.28 & $\begin{array}{l}1.21 \\
1.88\end{array}$ & 0.06 \\
\hline 964D-7H-1 & 64.32 & 1.78 & -0.23 \\
\hline $964 \mathrm{~A}-7 \mathrm{H}-1$ & 64.73 & 1.25 & 0.10 \\
\hline $964 \mathrm{~A}-7 \mathrm{H}-1$ & 64.83 & 1.14 & 0.19 \\
\hline & & & \\
\hline
\end{tabular}

\begin{tabular}{|c|c|c|c|}
\hline $\begin{array}{l}\text { Hole, core, } \\
\text { section }\end{array}$ & $\begin{array}{l}\text { Depth } \\
\text { (rmcd) }\end{array}$ & $\begin{array}{c}\delta^{18} \mathrm{O} \\
(\%, \mathrm{PDB})\end{array}$ & $\begin{array}{c}\delta^{13} \mathrm{C} \\
(\% o, \text { PDB })\end{array}$ \\
\hline $964 \mathrm{~A}-7 \mathrm{H}-2$ & 65.18 & 1.46 & 0.68 \\
\hline $964 \mathrm{~A}-7 \mathrm{H}-2$ & 65.18 & 1.49 & 0.80 \\
\hline $964 \mathrm{~A}-7 \mathrm{H}-2$ & 65.62 & 1.59 & -0.33 \\
\hline $964 \mathrm{~A}-7 \mathrm{H}-2$ & 65.81 & 1.79 & 0.49 \\
\hline $964 \mathrm{~A}-7 \mathrm{H}-2$ & 66.02 & 1.46 & -0.16 \\
\hline $964 \mathrm{~A}-7 \mathrm{H}-2$ & 66.17 & 1.35 & -0.28 \\
\hline $964 \mathrm{~A}-7 \mathrm{H}-2$ & 66.37 & 1.79 & $\begin{array}{l}-0.2 \mathrm{O} \\
-0.05\end{array}$ \\
\hline $964 \mathrm{~A}-7 \mathrm{H}-3$ & 66.48 & 2.09 & -0.17 \\
\hline $964 \mathrm{~A}-7 \mathrm{H}-3$ & 66.68 & 1.94 & -0.38 \\
\hline $964 \mathrm{~A}-7 \mathrm{H}-3$ & 66.86 & 2.22 & -0.11 \\
\hline 964D-7H-4 & 67.64 & 1.86 & -0.46 \\
\hline 964D-7H-4 & 67.84 & 1.35 & -0.72 \\
\hline $964 \mathrm{D}-7 \mathrm{H}-4$ & 68.04 & 1.46 & -0.46 \\
\hline 964D-7H-4 & 68.26 & 1.34 & 0.03 \\
\hline $964 \mathrm{D}-7 \mathrm{H}-4$ & 68.26 & 1.29 & -0.40 \\
\hline $964 \mathrm{D}-7 \mathrm{H}-4$ & 68.44 & 0.99 & -1.03 \\
\hline 964D-7H-4 & 68.62 & 1.65 & -0.20 \\
\hline 964D-7H-4 & 68.82 & 1.15 & -0.31 \\
\hline 964D-7H-5 & $\begin{array}{l}0.02 \\
68.92\end{array}$ & 1.12 & $\begin{array}{l}-0.31 \\
-0.35\end{array}$ \\
\hline 964D-7H-5 & 69.16 & 1.18 & -0.48 \\
\hline $964 \mathrm{D}-7 \mathrm{H}-5$ & 69.20 & 1.33 & -0.64 \\
\hline 964D-7H-5 & 69.36 & 1.84 & -0.72 \\
\hline $964 \mathrm{~B}-7 \mathrm{H}-2$ & 69.52 & 2.18 & -0.60 \\
\hline $964 \mathrm{~B}-7 \mathrm{H}-2$ & 69.52 & 2.01 & -0.82 \\
\hline $964 \mathrm{~B}-7 \mathrm{H}-2$ & 69.72 & 1.83 & -0.48 \\
\hline $964 \mathrm{~B}-7 \mathrm{H}-2$ & 69.72 & 2.02 & -0.68 \\
\hline $964 \mathrm{~B}-7 \mathrm{H}-2$ & 69.92 & 2.01 & 0.04 \\
\hline $964 \mathrm{~B}-7 \mathrm{H}-2$ & 70.12 & 1.90 & -0.04 \\
\hline $964 \mathrm{~B}-7 \mathrm{H}-2$ & 70.12 & 1.73 & -0.15 \\
\hline $964 \mathrm{~B}-7 \mathrm{H}-2$ & 70.30 & 1.49 & -0.26 \\
\hline $964 \mathrm{~B}-7 \mathrm{H}-3$ & 70.42 & 1.25 & $\begin{array}{r}-0.20 \\
0.00\end{array}$ \\
\hline 964B-7H-3 & 70.42 & 1.24 & -0.16 \\
\hline $964 \mathrm{~B}-7 \mathrm{H}-3$ & 70.42 & 1.34 & -0.21 \\
\hline 964B-7H-4 & 72.92 & 1.17 & -0.37 \\
\hline $964 \mathrm{~B}-7 \mathrm{H}-4$ & 73.12 & 1.62 & -0.14 \\
\hline $964 \mathrm{~B}-7 \mathrm{H}-4$ & 73.31 & $\begin{array}{l}1.74 \\
\end{array}$ & -0.34 \\
\hline $964 \mathrm{~B}-7 \mathrm{H}-5$ & 73.43 & 1.31 & -0.40 \\
\hline $964 \mathrm{~B}-7 \mathrm{H}-5$ & 73.64 & 1.28 & -0.50 \\
\hline $964 \mathrm{~B}-7 \mathrm{H}-5$ & 73.64 & 1.36 & -0.35 \\
\hline 964B-7H-5 & 73.86 & 1.10 & -0.61 \\
\hline $964 \mathrm{~B}-7 \mathrm{H}-5$ & 73.86 & 1.31 & -0.44 \\
\hline $964 \mathrm{E}-4 \mathrm{H}-2$ & 74.51 & 1.31 & -0.38 \\
\hline $964 \mathrm{~B}-7 \mathrm{H}-5$ & 74.51 & 1.06 & $\begin{array}{l}-0.30 \\
-0.35\end{array}$ \\
\hline $964 \mathrm{~B}-7 \mathrm{H}-5$ & 74.52 & 1.39 & -0.06 \\
\hline $964 \mathrm{E}-4 \mathrm{H}-2$ & 75.17 & 1.01 & -0.36 \\
\hline $964 \mathrm{E}-4 \mathrm{H}-3$ & 75.70 & 1.71 & -0.81 \\
\hline $964 \mathrm{E}-4 \mathrm{H}-3$ & 75.91 & 1.55 & -1.18 \\
\hline $964 \mathrm{E}-4 \mathrm{H}-3$ & 76.14 & 1.62 & -0.50 \\
\hline $964 \mathrm{E}-4 \mathrm{H}-3$ & 76.32 & $\begin{array}{l}1.02 \\
1.51\end{array}$ & $\begin{array}{l}-0.41 \\
0.41\end{array}$ \\
\hline $964 \mathrm{E}-4 \mathrm{H}-3$ & 76.52 & 1.28 & 0.50 \\
\hline $964 \mathrm{E}-4 \mathrm{H}-3$ & 76.73 & 1.45 & 0.09 \\
\hline $964 \mathrm{E}-4 \mathrm{H}-4$ & 77.62 & 1.67 & -0.50 \\
\hline $964 \mathrm{E}-4 \mathrm{H}-4$ & 77.82 & 2.05 & -0.60 \\
\hline $964 \mathrm{E}-4 \mathrm{H}-4$ & 78.01 & 1.24 & -0.21 \\
\hline $964 \mathrm{E}-4 \mathrm{H}-4$ & $\begin{array}{l}78.19 \\
78.19\end{array}$ & $\begin{array}{l}1.24 \\
1.09\end{array}$ & $\begin{array}{l}-0.21 \\
-0.23\end{array}$ \\
\hline $964 \mathrm{E}-4 \mathrm{H}-4$ & 78.37 & 0.88 & -0.52 \\
\hline $964 \mathrm{E}-4 \mathrm{H}-5$ & 78.64 & 1.37 & 0.01 \\
\hline $964 \mathrm{E}-4 \mathrm{H}-5$ & 78.84 & 1.26 & -0.28 \\
\hline $964 \mathrm{E}-4 \mathrm{H}-5$ & 79.20 & 1.80 & 0.10 \\
\hline $964 \mathrm{E}-4 \mathrm{H}-5$ & 79.37 & $\begin{array}{l}1.58 \\
\text {. }\end{array}$ & -0.25 \\
\hline $964 \mathrm{E}-4 \mathrm{H}-5$ & 79.55 & 1.07 & 0.01 \\
\hline $964 \mathrm{E}-4 \mathrm{H}-6$ & 79.85 & 1.58 & 0.05 \\
\hline $964 \mathrm{E}-4 \mathrm{H}-6$ & 80.03 & 1.46 & -0.45 \\
\hline $964 \mathrm{E}-4 \mathrm{H}-6$ & 80.21 & 1.54 & -0.54 \\
\hline $964 \mathrm{C}-8 \mathrm{H}-2$ & 80.36 & 1.88 & -0.25 \\
\hline $964 \mathrm{C}-8 \mathrm{H}-2$ & 80.52 & $\begin{array}{l}1.39 \\
1.39\end{array}$ & 0.40 \\
\hline $964 \mathrm{C}-8 \mathrm{H}-2$ & 80.69 & 1.05 & -0.17 \\
\hline $964 \mathrm{C}-8 \mathrm{H}-2$ & 80.87 & 1.29 & -0.13 \\
\hline $964 \mathrm{C}-8 \mathrm{H}-2$ & 81.04 & 1.66 & 0.38 \\
\hline $964 \mathrm{C}-8 \mathrm{H}-2$ & 81.20 & 1.72 & 0.97 \\
\hline $964 \mathrm{C}-8 \mathrm{H}-3$ & 81.68 & 1.20 & -0.22 \\
\hline $964 \mathrm{C}-8 \mathrm{H}-3$ & 82.11 & 1.07 & 0.33 \\
\hline $964 \mathrm{C}-8 \mathrm{H}-4$ & 82.61 & 1.66 & -0.34 \\
\hline $964 \mathrm{C}-8 \mathrm{H}-4$ & 82.80 & 1.21 & 0.74 \\
\hline $964 \mathrm{C}-8 \mathrm{H}-4$ & 82.97 & $\begin{array}{l}1.59 \\
1.59\end{array}$ & 0.25 \\
\hline $964 \mathrm{C}-8 \mathrm{H}-4$ & 83.15 & 1.81 & -0.40 \\
\hline $964 \mathrm{C}-8 \mathrm{H}-4$ & 83.33 & 1.09 & -0.29 \\
\hline $964 \mathrm{C}-8 \mathrm{H}-4$ & 83.51 & 1.52 & 0.17 \\
\hline $964 \mathrm{C}-8 \mathrm{H}-4$ & 83.70 & 1.10 & -0.18 \\
\hline $964 \mathrm{C}-8 \mathrm{H}-4$ & 83.88 & 1.29 & -0.34 \\
\hline $964 \mathrm{C}-8 \mathrm{H}-4$ & 83.88 & 1.10 & -0.47 \\
\hline $964 \mathrm{C}-8 \mathrm{H}-5$ & 84.00 & 1.65 & -0.89 \\
\hline $964 \mathrm{E}-5 \mathrm{H}-2$ & 84.33 & 2.04 & -0.52 \\
\hline $964 \mathrm{E}-5 \mathrm{H}-2$ & 84.50 & 1.49 & -0.23 \\
\hline $964 \mathrm{E}-5 \mathrm{H}-2$ & 84.74 & 1.51 & -0.42 \\
\hline $964 \mathrm{E}-5 \mathrm{H}-2$ & 84.94 & 1.47 & -0.59 \\
\hline $964 \mathrm{E}-5 \mathrm{H}-2$ & 85.32 & 1.95 & -1.06 \\
\hline & & & \\
\hline
\end{tabular}


Table 5 (continued).

\begin{tabular}{|c|c|c|c|}
\hline $\begin{array}{l}\text { Hole, core, } \\
\text { section }\end{array}$ & $\begin{array}{l}\text { Depth } \\
\text { (rmcd) }\end{array}$ & $\begin{array}{c}\delta^{18} \mathrm{O} \\
(\% o, \mathrm{PDB})\end{array}$ & $\begin{array}{c}\delta^{13} \mathrm{C} \\
(\%, \mathrm{PDB})\end{array}$ \\
\hline $964 \mathrm{E}-5 \mathrm{H}-2$ & 85.72 & 2.14 & -0.37 \\
\hline $964 \mathrm{E}-5 \mathrm{H}-3$ & 86.32 & 1.69 & -0.70 \\
\hline $964 \mathrm{E}-5 \mathrm{H}-3$ & 86.59 & 1.08 & -0.59 \\
\hline $964 \mathrm{E}-5 \mathrm{H}-3$ & 87.06 & 1.32 & 0.07 \\
\hline $964 \mathrm{E}-5 \mathrm{H}-3$ & 87.27 & 1.42 & -0.20 \\
\hline $964 \mathrm{E}-5 \mathrm{H}-3$ & 87.47 & 1.33 & -0.53 \\
\hline $964 \mathrm{E}-5 \mathrm{H}-4$ & 87.59 & 1.18 & -0.36 \\
\hline 964E-5H-4 & 87.78 & 1.12 & -0.70 \\
\hline $964 \mathrm{E}-5 \mathrm{H}-4$ & 87.98 & 0.85 & -0.47 \\
\hline $964 \mathrm{C}-9 \mathrm{H}-1$ & 88.79 & 0.58 & -1.12 \\
\hline $964 \mathrm{C}-9 \mathrm{H}-1$ & 89.02 & 1.33 & -0.73 \\
\hline $964 \mathrm{C}-9 \mathrm{H}-1$ & 89.22 & 1.49 & -0.53 \\
\hline $964 \mathrm{C}-9 \mathrm{H}-1$ & 89.42 & 1.57 & 0.14 \\
\hline $964 \mathrm{C}-9 \mathrm{H}-1$ & 89.62 & 1.63 & -0.18 \\
\hline $964 \mathrm{C}-9 \mathrm{H}-1$ & 89.62 & 1.60 & -0.41 \\
\hline $964 \mathrm{C}-9 \mathrm{H}-1$ & 89.80 & 1.15 & -0.18 \\
\hline $964 \mathrm{C}-9 \mathrm{H}-2$ & 89.92 & 1.14 & 0.22 \\
\hline $964 \mathrm{C}-9 \mathrm{H}-2$ & 90.16 & 0.93 & -0.31 \\
\hline $964 \mathrm{C}-9 \mathrm{H}-2$ & 90.32 & 1.28 & -0.59 \\
\hline $964 \mathrm{C}-9 \mathrm{H}-2$ & 90.72 & 0.97 & -0.39 \\
\hline $964 \mathrm{C}-9 \mathrm{H}-2$ & 90.92 & 0.86 & -0.04 \\
\hline $964 \mathrm{C}-9 \mathrm{H}-2$ & 91.12 & 0.97 & -0.73 \\
\hline $964 \mathrm{C}-9 \mathrm{H}-2$ & 91.30 & 1.53 & -0.49 \\
\hline $964 \mathrm{C}-9 \mathrm{H}-3$ & 91.42 & 1.39 & -0.67 \\
\hline $964 \mathrm{C}-9 \mathrm{H}-3$ & 91.62 & 1.19 & -0.92 \\
\hline $964 \mathrm{C}-9 \mathrm{H}-3$ & 91.82 & 1.45 & -0.29 \\
\hline $964 \mathrm{C}-9 \mathrm{H}-3$ & 92.02 & 1.15 & -0.62 \\
\hline $964 \mathrm{C}-9 \mathrm{H}-5$ & 94.82 & 1.81 & -0.32 \\
\hline $964 \mathrm{C}-9 \mathrm{H}-5$ & 95.04 & 1.60 & -0.23 \\
\hline $964 \mathrm{C}-9 \mathrm{H}-5$ & 95.14 & 1.59 & -0.47 \\
\hline $964 \mathrm{C}-9 \mathrm{H}-5$ & 95.28 & 1.14 & 0.08 \\
\hline $964 \mathrm{C}-9 \mathrm{H}-5$ & 97.27 & 0.88 & -0.09 \\
\hline $964 \mathrm{C}-9 \mathrm{H}-5$ & 97.44 & 0.92 & -0.13 \\
\hline $964 \mathrm{C}-9 \mathrm{H}-6$ & 97.55 & 1.29 & 0.15 \\
\hline $964 \mathrm{C}-9 \mathrm{H}-6$ & 97.75 & 1.35 & 0.40 \\
\hline $964 \mathrm{C}-9 \mathrm{H}-6$ & 98.09 & 0.58 & 0.54 \\
\hline $964 \mathrm{E}-6 \mathrm{H}-2$ & 98.38 & 1.51 & 0.60 \\
\hline $964 \mathrm{E}-6 \mathrm{H}-2$ & 98.38 & 1.50 & 0.52 \\
\hline $964 \mathrm{E}-6 \mathrm{H}-2$ & 98.47 & 1.47 & 0.42 \\
\hline $964 \mathrm{E}-6 \mathrm{H}-2$ & 98.60 & 1.53 & 0.46 \\
\hline $964 \mathrm{E}-6 \mathrm{H}-2$ & 98.83 & 1.11 & 0.14 \\
\hline $964 \mathrm{E}-6 \mathrm{H}-2$ & 98.83 & 1.39 & 0.12 \\
\hline $964 \mathrm{E}-6 \mathrm{H}-2$ & 99.04 & 0.83 & -0.29 \\
\hline $964 \mathrm{E}-6 \mathrm{H}-2$ & 99.04 & 1.01 & 0.23 \\
\hline $964 \mathrm{E}-6 \mathrm{H}-2$ & 99.24 & 1.27 & 0.09 \\
\hline $964 \mathrm{E}-6 \mathrm{H}-2$ & 99.44 & 1.37 & -0.21 \\
\hline $964 \mathrm{E}-6 \mathrm{H}-2$ & 99.44 & 1.40 & -0.35 \\
\hline $964 \mathrm{E}-6 \mathrm{H}-3$ & 99.56 & 1.33 & -0.29 \\
\hline $964 \mathrm{E}-6 \mathrm{H}-3$ & 99.79 & 1.17 & -0.47 \\
\hline $964 \mathrm{E}-6 \mathrm{H}-3$ & 100.48 & 1.14 & 0.25 \\
\hline $964 \mathrm{E}-6 \mathrm{H}-3$ & 100.70 & 1.22 & -0.95 \\
\hline $964 \mathrm{E}-6 \mathrm{H}-3$ & 100.86 & 1.02 & -0.65 \\
\hline $964 \mathrm{E}-6 \mathrm{H}-4$ & 101.21 & 0.97 & -0.22 \\
\hline $964 \mathrm{E}-6 \mathrm{H}-4$ & 101.42 & 0.84 & -0.35 \\
\hline $964 \mathrm{E}-6 \mathrm{H}-4$ & 101.85 & 1.06 & -0.77 \\
\hline 964E-6H-4 & 102.09 & 0.74 & -0.68 \\
\hline $964 \mathrm{E}-6 \mathrm{H}-4$ & 102.24 & 0.55 & -0.29 \\
\hline $964 \mathrm{E}-6 \mathrm{H}-4$ & 102.56 & 1.08 & -0.26 \\
\hline $964 \mathrm{E}-6 \mathrm{H}-4$ & 102.78 & 1.27 & -0.36 \\
\hline $964 \mathrm{E}-6 \mathrm{H}-4$ & 102.78 & 1.16 & -0.35 \\
\hline $964 \mathrm{E}-6 \mathrm{H}-5$ & 102.95 & 1.08 & -0.79 \\
\hline $964 \mathrm{E}-6 \mathrm{H}-5$ & 103.43 & 0.82 & -0.51 \\
\hline
\end{tabular}

No overall long-term trends can be seen in the carbon isotope records at Site 963 (Fig. 6) or Site 964 (Fig. 7). At both sites, fluctuations between periods of isotopic enrichment and depletion can be seen in the $\delta^{13} \mathrm{C}$ records of $G$. bulloides. The G. bulloides $\delta^{13} \mathrm{C}$ record at Site 963 indicates that for at least the past $1.25 \mathrm{Ma}$, values have been lower than those reported for this species in the present-day Mediterranean (Fig. 6). This implies that the surface waters of the Strait of Sicily have been either (1) more productive and/or nutrient rich than the other sections of the Mediterranean or (2) that a shoaling of a deeper water mass relatively rich in nutrients into the photic zone and increased surface-water stratification occurred throughout most of the last $1.5 \mathrm{~m}$.y. at this site. However, the lower mean $\delta^{13} \mathrm{C}$ of $G$. bulloides at Site 963 through this time period may also reflect the increased input of isotopically lighter terrestrial organic matter into the surface waters as a result of increased land-derived sedimentation at this site. Vergnaud-Grazzini (1983) has noted that near continents, the $\delta^{13} \mathrm{C}$ of the surface-water $\Sigma \mathrm{CO}_{2}$ is affected by runoff, which trans-

\begin{tabular}{|c|c|c|c|}
\hline $\begin{array}{l}\text { Hole, core, } \\
\text { section }\end{array}$ & $\begin{array}{l}\text { Depth } \\
\text { (rmcd) }\end{array}$ & $\begin{array}{c}\delta^{18} \mathrm{O} \\
(\% o, \mathrm{PDB})\end{array}$ & $\begin{array}{c}\delta^{13} \mathrm{C} \\
(\%, \mathrm{PDB})\end{array}$ \\
\hline 964E-6H-5 & 103.63 & 0.54 & -0.79 \\
\hline $964 \mathrm{E}-6 \mathrm{H}-5$ & 104.04 & 0.90 & -0.81 \\
\hline 964E-6H-5 & 104.24 & 1.07 & -0.45 \\
\hline $964 \mathrm{E}-6 \mathrm{H}-5$ & 104.42 & 0.73 & -0.53 \\
\hline $964 \mathrm{E}-6 \mathrm{H}-6$ & 104.72 & 0.86 & -0.40 \\
\hline $964 \mathrm{E}-6 \mathrm{H}-6$ & 104.96 & 0.61 & -0.10 \\
\hline $964 \mathrm{E}-6 \mathrm{H}-6$ & 105.16 & 0.83 & -0.39 \\
\hline 964B-10-1 & 105.27 & 0.88 & -0.68 \\
\hline $964 \mathrm{~B}-10 \mathrm{H}-1$ & 105.27 & 0.69 & -0.39 \\
\hline 964B-10H-1 & 105.27 & 0.94 & -0.21 \\
\hline $964 \mathrm{E}-6 \mathrm{H}-6$ & 105.40 & 0.92 & -0.62 \\
\hline $964 \mathrm{E}-6 \mathrm{H}-6$ & 105.40 & 0.98 & -0.43 \\
\hline 964B-10H-1 & 105.46 & 0.90 & -0.36 \\
\hline 964B-10H-1 & 105.65 & 0.61 & -0.80 \\
\hline 964B-10H-1 & 105.84 & 0.95 & -0.74 \\
\hline 964B-10H-1 & 106.07 & 0.84 & -0.72 \\
\hline $964 \mathrm{~B}-10 \mathrm{H}-3$ & 108.30 & 0.77 & -0.70 \\
\hline 964B-10H-3 & 108.50 & 0.41 & -0.67 \\
\hline $964 \mathrm{~B}-10 \mathrm{H}-3$ & 108.91 & 0.45 & -0.58 \\
\hline $964 \mathrm{~B}-10 \mathrm{H}-3$ & 109.07 & 0.53 & 0.07 \\
\hline $964 \mathrm{~B}-10 \mathrm{H}-3$ & 109.22 & 0.87 & -0.34 \\
\hline $964 \mathrm{~B}-10 \mathrm{H}-3$ & 109.40 & 0.66 & -0.07 \\
\hline 964B-10H-4 & 109.52 & 0.52 & -0.34 \\
\hline $964 \mathrm{~B}-10 \mathrm{H}-4$ & 109.92 & 0.72 & -0.01 \\
\hline $964 \mathrm{~B}-10 \mathrm{H}-4$ & 110.12 & 0.64 & -0.24 \\
\hline 964B-10H-4 & 110.32 & 0.56 & -0.36 \\
\hline $964 \mathrm{~B}-10 \mathrm{H}-4$ & 110.52 & 0.83 & -0.27 \\
\hline $964 \mathrm{~B}-10 \mathrm{H}-4$ & 110.52 & 0.54 & -0.37 \\
\hline 964B-10H-4 & 110.72 & 0.92 & -0.30 \\
\hline $964 \mathrm{~B}-10 \mathrm{H}-4$ & 110.90 & 1.03 & -0.28 \\
\hline 964B-10H-5 & 111.02 & 0.78 & -0.05 \\
\hline $964 \mathrm{~B}-10 \mathrm{H}-5$ & 111.22 & 0.84 & -0.19 \\
\hline $964 \mathrm{~B}-10 \mathrm{H}-5$ & 111.42 & 0.65 & -0.39 \\
\hline 964B-10H-5 & 111.62 & 0.86 & -0.11 \\
\hline $964 \mathrm{~B}-10 \mathrm{H}-5$ & 111.82 & 1.01 & -0.20 \\
\hline 964B-10H-5 & 112.03 & 0.96 & -0.24 \\
\hline $964 \mathrm{~B}-10 \mathrm{H}-5$ & 112.03 & 0.93 & -0.37 \\
\hline $964 \mathrm{~B}-10 \mathrm{H}-5$ & 112.24 & 1.11 & -0.46 \\
\hline 964B-10H-5 & 112.24 & 0.80 & -0.47 \\
\hline $964 \mathrm{~B}-10 \mathrm{H}-5$ & 112.41 & 0.96 & -0.60 \\
\hline $964 \mathrm{~B}-10 \mathrm{H}-6$ & 112.52 & 1.00 & -0.29 \\
\hline 964B-10H-6 & 112.70 & 0.84 & -0.49 \\
\hline $964 \mathrm{~A}-10 \mathrm{H}-5$ & 112.78 & 0.76 & -0.61 \\
\hline 964B-10H-6 & 112.88 & 0.86 & -0.56 \\
\hline $964 \mathrm{~A}-10 \mathrm{H}-5$ & 112.98 & 0.92 & -0.62 \\
\hline $964 \mathrm{~A}-10 \mathrm{H}-5$ & 113.16 & 1.25 & -0.52 \\
\hline 964B-10H-6 & 113.30 & 1.22 & -0.70 \\
\hline $964 \mathrm{~B}-10 \mathrm{H}-6$ & 113.49 & 1.15 & -0.62 \\
\hline 964B-10H-6 & 113.69 & 1.04 & -0.30 \\
\hline $964 \mathrm{~B}-10 \mathrm{H}-6$ & 113.91 & 1.05 & -0.68 \\
\hline $964 \mathrm{~B}-10 \mathrm{H}-6$ & 114.09 & 1.00 & -0.34 \\
\hline 964B-10H-6 & 114.29 & 0.83 & -0.38 \\
\hline 964D-11H-1 & 114.97 & 0.74 & 0.22 \\
\hline 964D-11H-1 & 115.14 & 0.78 & -0.41 \\
\hline 964D-11H-1 & 115.52 & 0.93 & -0.88 \\
\hline $964 \mathrm{D}-11 \mathrm{H}-1$ & 115.70 & 0.99 & -0.62 \\
\hline 964D-11H-1 & 115.95 & 1.00 & -0.50 \\
\hline 964D-11H-1 & 116.19 & 0.80 & -0.25 \\
\hline 964D-11H-2 & 117.16 & 0.85 & -0.26 \\
\hline 964D-12H-5 & 131.02 & 0.68 & -0.44 \\
\hline 964D-12H-5 & 131.17 & 0.80 & -0.13 \\
\hline
\end{tabular}

ports meteoric waters enriched in terrestrial compounds with low $\delta^{13} \mathrm{C}$ values. This may also explain the apparent difference in the $\delta^{13} \mathrm{C}$ of $G$. bulloides between the sites featured in this study, as the $\delta^{13} \mathrm{C}$ values of this species at Site 963 are on the average lower than Site 964 (Table 7).

The difference between modern and fossil $\delta^{13} \mathrm{C}$ values of $G$. bulloides from Site 964 exhibits distinct patterns within two depth-time intervals. Between 120 and $60 \mathrm{rmcd}(\sim 1.9 \mathrm{Ma}), \delta^{13} \mathrm{C}$ values are generally higher than the modern average, with lower values occurring during the formation of sapropels (Fig. 7). Between $60 \mathrm{rmcd}(\sim 1.9$ $\mathrm{Ma}$ ) and the top of the Site 964 composite section ( 0.18 Ma), G. bulloides exhibits a higher frequency of $\delta^{13} \mathrm{C}$ values lower than the modern-day average (Fig. 7). As in the preceding interval, minimum $\delta^{13} \mathrm{C}$ values are generally associated with sapropels (see the following section, "Sapropels," for further discussion). This suggests that the intensification of productivity through the input of nutrients into the photic zone, surface-water stratification, and/or input of terrestrial or- 


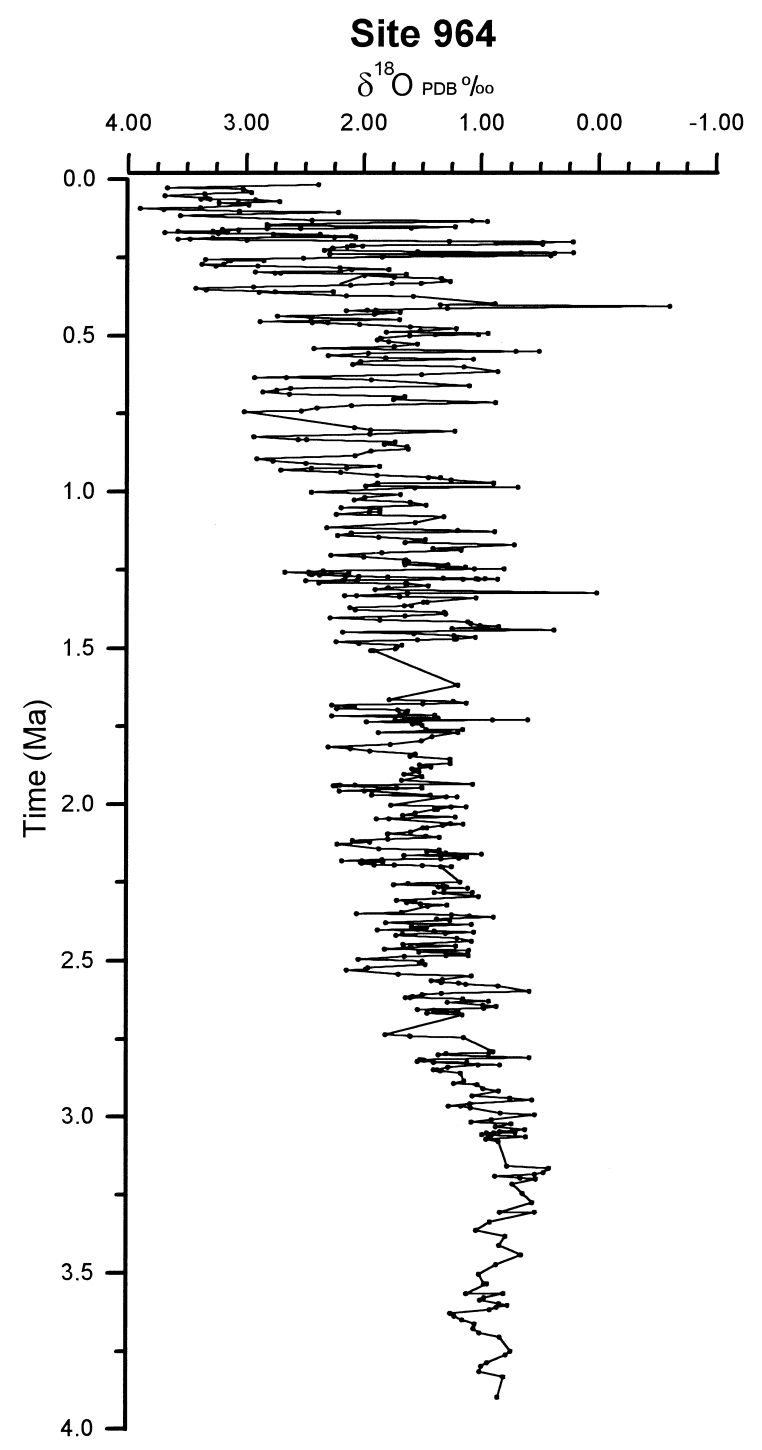

Figure 5. Site 964 oxygen isotope record of G. bulloides plotted vs. time.

Table 6. Site 964 oxygen isotope averages ( $G$, bulloides).

\begin{tabular}{lcc}
\hline $\begin{array}{c}\text { Time interval } \\
(\mathrm{Ma})\end{array}$ & $\begin{array}{c}\text { Mean } \delta^{18} \mathrm{O} \\
(\% \circ)\end{array}$ & $\begin{array}{c}\delta^{18} \mathrm{O} \text { avg. } \\
\text { deviation }(\% \circ)\end{array}$ \\
\hline Recent-0.47 & 2.53 & 0.60 \\
$0.47-0.95$ & 2.03 & 0.44 \\
$0.95-1.51$ & 1.75 & 0.36 \\
$1.51-2.10$ & 1.61 & 0.27 \\
$2.10-2.60$ & 1.49 & 0.27 \\
$2.60-3.20$ & 1.07 & 0.27 \\
$3.20-3.60$ & 0.82 & 0.15 \\
$3.60-3.90$ & 0.92 & 0.13 \\
\hline
\end{tabular}

ganic matter may have intensified as a result of climatic cooling during the Pleistocene.

\section{SAPROPELS}

A distinguishing feature of the oxygen isotope records from Sites 963 and 964 is the relatively large excursions that are associated with many of the sapropels at the sites. As discussed previously, these excursions (up to $3 \%$ ) have been previously observed by many workers

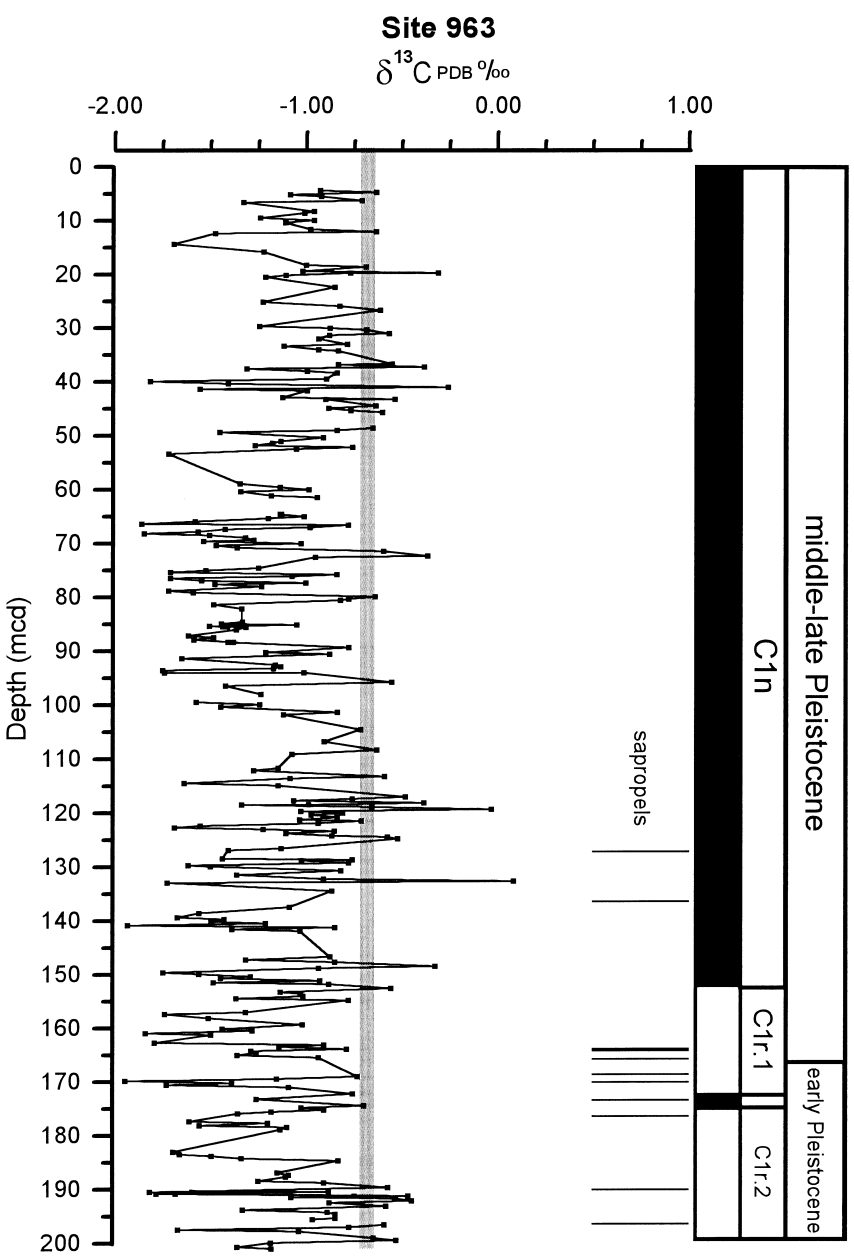

Figure 6. Site 963 carbon isotope record of G. bulloides plotted against composite depth (mcd). The stratigraphic positions of the paleomagnetic reversals are indicated on the right. The solid lines on the left mark the stratigraphic positions of the sapropels. The shaded area represents the present-day range of Mediterranean G. bulloides $\delta^{13} \mathrm{C}$ values.

(Emiliani, 1955, 1974; Williams et al., 1978; Vergnaud-Grazzini et al., 1977; Rossignol-Strick et al., 1982; Thunell et al., 1987; among others) and have been attributed to the reduction of surface-water salinities during sapropel formation rather than large increases in surface-water temperatures and decreasing ice volume (although certain sapropel intervals do not exhibit the characteristic large-amplitude decreases in the $\delta^{18} \mathrm{O}$ of $G$. bulloides). Oxygen isotope data for some sapropels were unavailable because of lack of sample material, and therefore the record may not reflect the true signal in these cases.

The $\delta^{18} \mathrm{O}$ record of $G$. bulloides from Site 963 indicates that not all reductions in surface-water salinities are accompanied by events of sapropel formation in the Strait of Sicily (Fig. 2). This is noteworthy, as the magnitude and (apparent) timing of the isotope anomalies are strikingly similar to those observed at Site 964, which contains a higher frequency of sapropels with greater organic carbon content (see Emeis, Robertson, Richter, et al., 1996, for Site 963 and Site 964 sapropel data). This suggests that surface-water hydrography played a different role in establishing the necessary surface-productivity and/or bottom-water reducing conditions at the two sites. This interpretation is reasonable, given that the difference in depth between the two sites is greater than $3 \mathrm{~km}$.

The frequency of (known) sapropel occurrence at Site 963 progressively decreases with decreasing age (Fig. 2). The highest fre- 


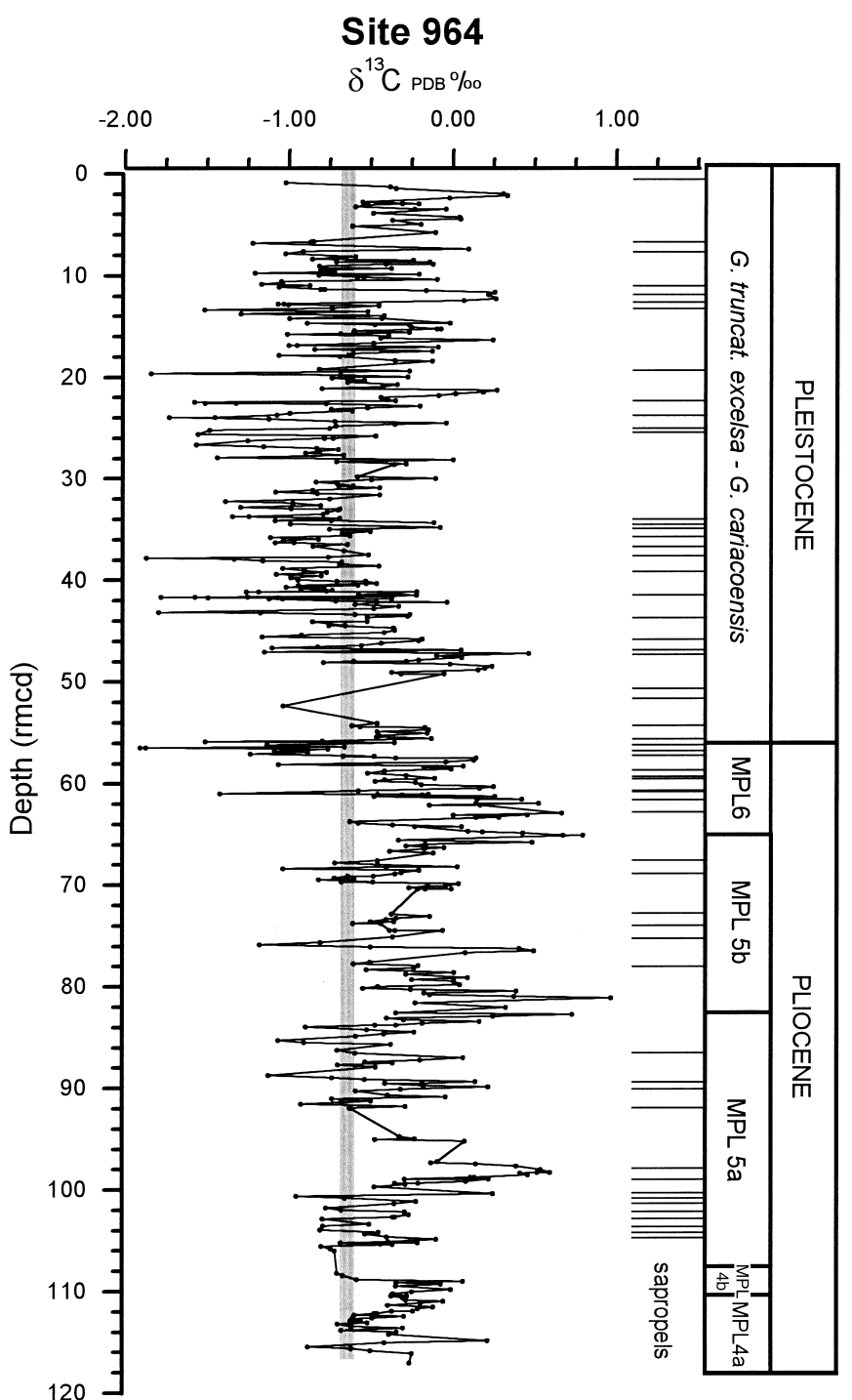

Figure 7. Site 964 carbon isotope record of G. bulloides plotted against revised composite depth (rmcd). The solid lines on the left mark the stratigraphic positions of the sapropels. The planktonic foraminiferal zonation scheme is after Cita (1975). The shaded area represents the present-day range of Mediterranean $G$. bulloides $\delta^{13} \mathrm{C}$ values.

Table 7. Mean $\delta^{13} \mathrm{C}$ values (G. bulloides).

\begin{tabular}{cc}
\hline Site 963 & Site 964 \\
\hline$-1.13 \% \circ$ & $-0.48 \% \circ$
\end{tabular}

quency of sapropels was found below the approximate level of the Cr.1r1 paleomagnetic event $(0.98 \mathrm{Ma})$, after which a major cooling event occurs. Only two sapropel events occur between the Brunhes/ Matuyama boundary and the last occurrence (LO) of the calcareous nannofossil $P$. lacunosa $(0.46 \mathrm{Ma})$ at $117 \mathrm{mcd}$. As previously discussed, the $\delta^{18} \mathrm{O}$ of $G$. bulloides at Site 963 indicates an additional cooling phase in the Mediterranean that is associated with the dominant 100-k.y. cycle of changes in global ice volume. The distribution of sapropels within the Site $963 \delta^{18} \mathrm{O}$ record of G. bulloides suggests that there may be an inverse relationship between the intensity of glaciations and the frequency of sapropel formation.
At Site 964 , the magnitude of the $\delta^{18} \mathrm{O}$ anomalies associated with sapropels generally increases with decreasing age (Fig. 5). This most likely reflects how this site responded to changes in the global climate regime. The impact of glaciation most likely intensified the seasonal contrasts in the local water balance as seen in the progressively larger amplitude shifts in the $\delta^{18} \mathrm{O}$ of $G$. bulloides with decreasing age.

As previously discussed, sapropels at Site 963 and Site 964 generally occur in intervals when $\delta^{13} \mathrm{C}$ values for $G$. bulloides are lower than present day and most likely reflect increased surface-water stratification and/or input of terrestrial organic matter or nutrients into surface waters (Figs. 6, 7). However, this is not meant to imply that sapropels are primarily the result of increased terrestrial organic matter into the Mediterranean. Previous studies (e.g., Sutherland et al., 1984; Smith et al., 1986; ten Haven et al., 1987) and shipboard analyses from this leg (Emeis, Robertson, Richter, et al., 1996) indicate that the organic matter in sapropels is primarily from a marine source. In addition, large increases in terrestrial organic carbon input would have most likely been accompanied by increases in nonorganic terrigenous sediments, thus resulting in the dilution of the organic carbon content. At Site 963, shipboard analyses of sapropels indicate the presence of marine organic matter under a regime of high rates of sedimentation (Emeis, Robertson, Richter, et al., 1996). Thunell et al. (1990) conclude that the low $\delta^{13} \mathrm{C}$ values exhibited by planktonic foraminifers from sapropels argue against the idea that sapropels are entirely the result of increased surface-water productivity (Calvert, 1983; Calvert et al., 1992). However, the organic carbon content of sapropels reported by Emeis, Robertson, Richter, et al. (1996) from Site 964 (up to 25\%) would be difficult to achieve without a major increase in productivity. In their study of the Bannock Basin, Eastern Mediterranean Sea, Howell and Thunell (1992) concluded that the presence of anoxic conditions alone, while sufficient to form sediments $\geq 2 \%$ organic carbon under current productivity levels, would have been insufficient to form a sapropel of higher organic content within a reasonable time frame. As shown in Figures 6 and 7, many of the sapropels from Site 963 and most of the sapropels from Site 964 were formed during periods where the $\delta^{13} \mathrm{C}$ values of $G$. bulloides were lower than the present-day average of $-0.7 \%$ o to $-0.6 \%$ o reported in Vergnaud-Grazzini et al. (1990). Our data support the concept that sapropels may have been formed during periods of surface-water stratification when shoaling of the nutricline into the photic zone fostered increased primary production, while the stratification of the surface waters enabled this layer to reside at shallower depths, resulting in decreased $\delta^{13} \mathrm{C}$ values of $G$. bulloides

A comparison of organic carbon data from selected sapropels from Hole 964A does not exhibit any systematic relationship between organic carbon richness and the $\delta^{13} \mathrm{C}$ of G. bulloides (Fig. 8). In this study we conclude that the $\delta^{13} \mathrm{C}$ cannot solely be used to estimate the magnitude of productivity increases and/or organic carbon sedimentation rates. However, isotopic analyses of other species in conjunction with $G$. bulloides may provide a more definitive assessment of these parameters.

No specific age assignments have been established for the Site 963 or Site 964 sapropels. At Site 964, linear interpolation of datums $<0.78 \mathrm{Ma}$ yields sapropel ages that are significantly different than those previously observed in the Mediterranean (e.g., Ryan, 1972; Lourens et al., 1996). As previously mentioned, the age models for both sites will require further revision before sapropel ages can be accurately determined.

\section{CONCLUSIONS}

An isotope stratigraphy based on analyses of $G$. bulloides has been developed for ODP Sites 963 and 964 . The isotope records from both sites provide an excellent record of major climate changes in the 


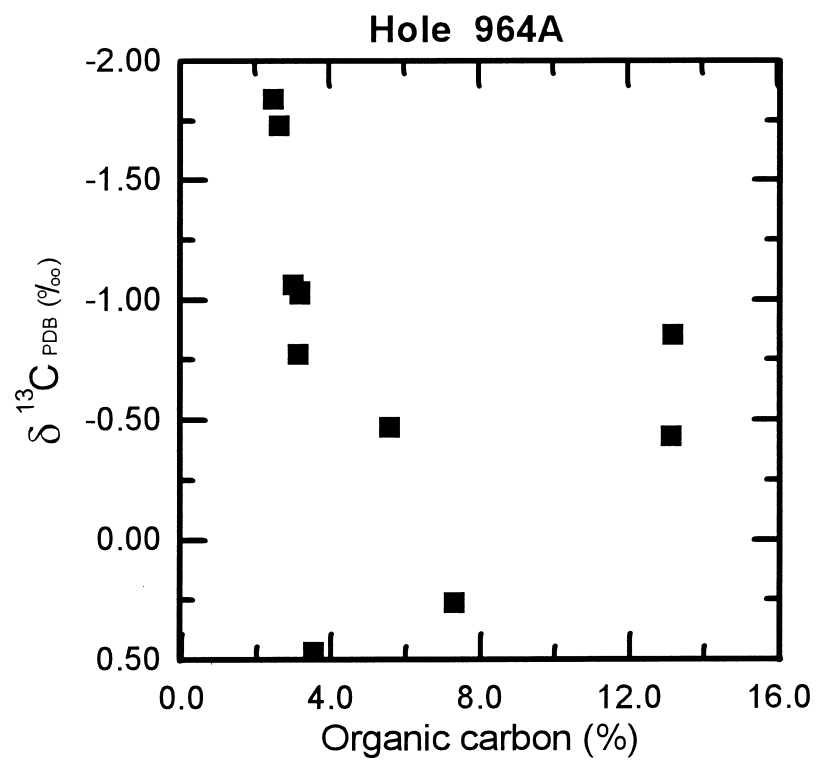

Figure 8. Organic carbon content vs. $\delta^{13} \mathrm{C}$ of $G$. bulloides from selected sapropels of Hole 964A.

Mediterranean. These changes were driven by global increases in ice volume and decreasing temperatures. The regional impact of these events can be seen in the high (glacial-interglacial) amplitude of the oxygen isotope records from both sites, which reflect reductions in Mediterranean surface-water salinities resulting from changes in the rates of evaporation/precipitation and fluvial runoff within the region. The formation of organic-rich sapropels is linked to these changes in the regional water budget, and the changes in surfacewater hydrography are associated with the development of bottomwater anoxia and/or enhanced productivity.

\section{ACKNOWLEDGMENTS}

We thank K. Cockrell, L. Coe, P. DuBois, C. Lewis, and J. Robinson for their assistance in the preparation of the sample material. We also thank L. Lourens and R. Tiedemann for their careful reviews of the manuscript. This work was supported by USSSP Award no. 160-F000194 to M. Howell.

\section{REFERENCES}

Anastasakis, G.C., and Stanley, D.J., 1986. Uppermost sapropel, Eastern Mediterranean: paleoceanography and stagnation. Nat. Geogr. Res., 2:179-197.

Backman, J., 1979. Pliocene biostratigraphy of DSDP Sites 111 and 116 from the North Atlantic Ocean and the age of Northern Hemisphere Glaciation. Stockholm Contrib. Geol., 32:115-137.

Béthoux, J.P., 1979. Budgets of the Mediterranean Sea: their dependence on the local climate and the characteristics of the Atlantic waters. Oceanol. Acta., 2:157-163.

- 1989. Oxygen consumption, new production, vertical advection and environmental evolution of the Mediterranean Sea. Deep-Sea Res., 36:769-781.

Bradley, W.H., 1938. Mediterranean sediments and Pleistocene sea levels. Science, 88:376-379.

Broecker, W.S., 1986. Oxygen isotope constraints on surface ocean temperatures. Quat. Res., 26:121-134.

Calvert, S.E., 1983. Geochemistry of Pleistocene sapropels and associated sediments from the Eastern Mediterranean. Oceanol. Acta, 6:225-267.

Calvert, S.E., Nielsen, B., and Fontugne, M.R., 1992. Evidence from nitrogen isotope ratios for enhanced productivity during the formation of eastern Mediterranean sapropels. Nature, 359:223-225.
Cande, S.C., and Kent, D.V., 1995. Revised calibration of the geomagnetic polarity timescale for the Late Cretaceous and Cenozoic. J. Geophys. Res., 100:6093-6095.

Castradori, D., 1993. Calcareous nannofossil biostratigraphy and biochronology in eastern Mediterranean deep-sea cores. Riv. Ital. Paleontol. Stratigr., 99:107-126.

Ciaranfi, N., and Cita, M.B., 1973. Paleontological evidence of changes in the Pliocene climates. In Ryan, W.B.F., and Hsü, K.J., et al., Init. Repts. DSDP, 13: Washington (U.S. Govt. Printing Office), 1387-1399.

Cita, M.B., 1975. Studi sul Pliocene e gli strati di passaggoi dal Miocene al Pliocene, VII. Planktonic foraminiferal biozonation of the Mediterranean Pliocene deep sea record: a revision. Riv. Ital. Paleontol. Stratigr, $81: 527-544$.

Cita, M.B., and Grignani, D., 1982. Nature and origin of Late Neogene Mediterranean sapropels. In Schlanger, S.O., and Cita, M.B. (Eds.), Nature and Origin of Cretaceous Carbon-rich Facies: London (Academic Press), 165-196.

Duplessy, J.C., 1972. La géochimie des isotopes stables du carbone dans la mer [Ph.D. dissert.]. Univ. of Paris.

Emeis, K.-C., Robertson, A.H.F., Richter, C., et al., 1996. Proc. ODP, Init. Repts., 160: College Station, TX (Ocean Drilling Program).

Emiliani, C., 1955. Pleistocene temperature variations in the Mediterranean. Quaternaria, 3:87-98.

, 1974. Isotopic paleotemperatures and shell morphology of Globingerinoides rubra in Mediterranean deep-sea core 189. Micropaleontology, 20:106-109.

Ganssen, G.M., and Troelstra, S.R., 1987. Paleoenvironmental change from stable isotopes in planktonic foraminifera from Eastern Mediterranean sapropels. Mar. Geol., 75:210-218.

Hilgen, F.J., 1991. Astronomical calibration of Gauss to Matuyama sapropels in the Mediterranean and implication for the geomagnetic polarity time scale. Earth Planet. Sci. Lett., 104:226-244.

Howell, M.W., Rio, D., and Thunell, R.C., 1990. Laminated sediments from the Vrica Section (Calabria, S. Italy): evidence for Plio-Pleistocene climatic change in the Mediterranean region. Palaeogeogr., Palaeoclimatol., Palaeoecol., 78:195-216.

Howell, M.W., and Thunell, R.C., 1992. Organic carbon accumulation in Bannock Basin: evaluating the role of productivity in the formation of Eastern Mediterranean sapropels. Mar. Geol., 103:461-471.

Hsü, K.J., Montadert, L., et al., 1978. Init. Repts. DSDP, 42 (Pt. 1): Washington (U.S. Govt. Printing Office).

Kastens, K.A., Mascle, J., Auroux, C., et al., 1987. Proc. ODP, Init. Repts., 107: College Station, TX (Ocean Drilling Program).

Keigwin., L.D., and Thunell, R.C., 1979. Middle Pliocene climatic change in the Western Mediterranean from faunal and oxygen isotopic trends. Nature, 282:292-296.

Lacombe, H., Gascard, J.C., Gonella, J., and Béthoux, J.P., 1981. Response of the Mediterranean to the water and energy fluxes across its surface, on seasonal and inter-annual scales. Oceanol. Acta, 4:247-255.

Lourens, L.J., Antonarakou, A., Hilgen, F.J., Van Hoof, A.A.M., VergnaudGrazzini, C., and Zachariasse, W.J., 1996. Evaluation of the Plio-Pleistocene astronomical timescale. Paleoceanography, 11:391-413.

Lourens, L.J., Hilgen, F.J., Gudjonsson, L., and Zachariasse, W.J., 1992. Late Pliocene to early Pleistocene astronomically forced sea surface productivity and temperature variations in the Mediterranean. Mar. Micropaleontol., 19:49-78.

Olausson, E., 1961. Studies of deep-sea cores. Rep. Swed. Deep-Sea Exped., 1947-1948, 8:335-391.

Pierre, C., Vergnaud-Grazzini, C., Thouron, D., and Saliège, J.F., 1986. Compositions isotopiques de l'oxygène et du carbone des masses d'eau en Méditerranée. Mem. Soc. Geol. Ital., 36:165-174.

Rio, D., Raffi, I., and Villa, G., 1990. Pliocene-Pleistocene calcareous nannofossil distribution patterns in the Western Mediterranean. In Kastens, K.A., Mascle, J., et al., Proc. ODP, Sci. Results, 107: College Station, TX (Ocean Drilling Program), 513-533.

Rio, D., Sprovieri, R., and Raffi, I., 1984. Calcareous plankton biostratigraphy and biochronology of the Pliocene-lower Pleistocene succession of the Capo Rossello area, Sicily. Mar. Micropaleontol., 9:135-180.

Rohling, E.J., 1991. Shoaling of the Eastern Mediterranean pycnocline due to reduction of excess evaporation: implications for sapropel formation. Paleoceanography, 6:537-541.

Rohling, E.J., and Gieskes, W.W.C., 1989. Late Quaternary changes in Mediterranean intermediate water density and formation rate. Paleoceanography, 4:531-545. 
Rossignol-Strick, M., Nesteroff, W., Olive, P., and Vergnaud-Grazzini, C., 1982. After the deluge: Mediterranean stagnation and sapropel formation. Nature, 295:105-110.

Ruddiman, W.F., McIntyre, A., and Raymo, M., 1987. Paleo-environmental results from North Atlantic Sites 607 and 609. In Ruddiman, W.F., Kidd, R.B., Thomas, E., et al., Init. Repts. DSDP, 94 (Pt. 2): Washington (U.S. Govt. Printing Office), 855-878.

Ruddiman, W.F., and Raymo, M.E., 1988. Northern hemisphere climatic regimes during the past 3 Ma: possible tectonic connections. Philos. Trans. R. Soc. London B, 318:411-430.

Ruddiman, W.F., Raymo, M.E., and McIntyre, A., 1986. Matuyama 41,000year cycles: North Atlantic Ocean and Northern Hemisphere ice sheets. Earth Planet. Sci. Lett., 80:117-129.

Ryan, W.B.F., 1972. Stratigraphy of late Quaternary sediments in the Eastern Mediterranean. In Stanley, D.J. (Ed.), The Mediterranean Sea: Stroudsburg, PA (Dowden, Hutchison and Ross), 149-169.

Ryan, W.B.F., Hsü, K.J., et al., 1973. Init. Repts. DSDP, 13 (Pts. 1 and 2): Washington (U.S. Govt. Printing Office)

Sarmiento, J., Herbert, T., and Toggweiler, J., 1988. Mediterranean nutrient balance and episodes of anoxia. Global Biogeochem. Cycles, 2:427-444.

Shackleton, N.J., Backman, J., Zimmerman, H., Kent, D.V., Hall, M.A., Roberts, D.G., Schnitker, D., Baldauf, J.G., Desprairies, A., Kaltenback, A.J., Krumsiek, K.A.O., Morton, A.C., Murray, J.W., and Westberg-Smith, J., 1984. Oxygen isotope calibration of the onset of ice-rafting and the history of glaciation in the North Atlantic region. Nature, 307:620-623.

Shackleton, N.J., and Hall, M.A., 1984. Oxygen and carbon isotope stratigraphy of Deep Sea Drilling Project Hole 552A: Plio-Pleistocene glacial history. In Roberts, D.G., Schnitker, D., et al., Init. Repts. DSDP, 81: Washington (U.S. Govt. Printing Office), 599-609.

Shackleton, N.J., Hall, M.A., and Pate, D., 1995. Pliocene stable isotope stratigraphy of Site 846. In Pisias, N.G., Mayer, L.A., Janecek, T.R., Palmer-Julson, A., and van Andel, T.H. (Eds.), Proc. ODP, Sci. Results, 138: College Station, TX (Ocean Drilling Program), 337-355.

Shackleton, N.J., and Opdyke, N.D., 1976. Oxygen-isotope and paleomagnetic stratigraphy of Pacific Core V28-239: late Pliocene to latest Pleistocene. In Cline, R.M., and Hays, J.D. (Eds.), Investigations of Late Quaternary Paleoceanography and Paleoclimatology. Mem.-Geol. Soc. Am., 145:449-464.

Smith, D.J., Eglinton, G., and Morris, R.J., 1986. The lipid geochemistry of a recent sapropel and associated sediments from the Hellenic Outer Ridge, eastern Mediterranean Sea. Philos. Trans. R. Soc. London A, 319:375419.

Sprovieri, R., 1993. Pliocene-early Pleistocene astronomically forced planktonic foraminifera abundance fluctuations and chronology of Mediterranean calcareous plankton bio-events. Riv. Ital. Paleontol. Stratigr., 99:371-414.

Sprovieri, R., Thunell, R., and Howell, M., 1986. Paleontological and geochemical analysis of three laminated sedimentary units of late Pliocene-early Pleistocene age from the Monte San Nicola section in Sicily. Riv. Ital. Paleontol. Stratigr.,92:401-434.

Stanley, D.J., Maldonado, A., and Stuckenrath, R., 1975. Strait of Sicily depositional rates and patterns, and possible reversal of currents during the late Quaternary. Palaeogeogr., Palaeoclimatol., Palaeoecol., 18:279-291.

Suc, J.-P., 1984. Origin and evolution of the Mediterranean vegetation and climate in Europe. Nature, 307:429-432.

, 1986. Flores néogènes de Méditerranée occidental. Climat et paléogéographie. Bull. Cent. Rech. Explor-Prod. Elf-Aquitaine, 10:477-488.

Sutherland, H.E., Calvert, S.E., and Morris, J.R., 1984. Geochemical studies of the recent sapropel and associated sediment from the Hellenic Outer Ridge, eastern Mediterranean Sea, I. Mineralogy and chemical composition. Mar. Geol., 56:79-92.

ten Haven, H.L., Baas, M., Kroot, M., de Leeuw, J.W., Schenck, P.A., and Ebbing, J., 1987. Late Quaternary Mediterranean sapropels: III. Assessment of source of input and palaeotemperature as derived from biological markers. Geochim. Cosmochim. Acta, 51:803-810.

Thiede, J., 1978. A glacial Mediterranean. Nature, 276:680-683.

Thunell, R.C., 1978. Distribution of planktonic foraminifera in surface sediments of the Mediterranean Sea. Mar. Micropaleontol., 3:147-173.
1979. Climatic evolution of the Mediterranean Sea during the last 5.0 million years. Sediment. Geol., 23:67-79.

Thunell, R.C., and Reynolds, L.A., 1984. Sedimentation of planktonic foraminifera: seasonal changes in species flux in the Panama Basin. Micropaleontology, 30:243-262.

Thunell, R.C., and Williams, D.F., 1983. The step-wise development of Pliocene-Pleistocene paleoclimate and paleoceanographic conditions in the Mediterranean: oxygen isotope studies and DSDP Site 125 and 132. In Meulencamp, J.E. (Ed.), Reconstruction of Marine Paleoenvironments. Utrecht Micropaleontol. Bull., 30:111-127.

Thunell, R.C., Williams, D.F., and Cita, M.B., 1983. Glacial anoxia in the Eastern Mediterranean. J. Foraminifera. Res., 13:283-290.

Thunell, R.C., Williams, D.F., and Howell, M., 1987. Atlantic-Mediterranean water exchange during the late Neogene. Paleoceanography, 2:661-678.

Thunell, R.C., Williams, D.F., Rio, D., Raffi, I., and Sprovieri, R., 1985. Pliocene-Pleistocene history of the Mediterranean: results from deep sea and land-based marine records. Terra Cognita, 5:89.

Thunell, R., Williams, D., Tappa, E., Rio, D., and Raffi, I., 1990. PliocenePleistocene stable isotope record for Ocean Drilling Program Site 653, Tyrrhenian Basin: implications for paleoenvironmental history of the Mediterranean Sea. In Kastens, K.A., Mascle, J., et al., Proc. ODP, Sci. Results, 107: College Station, TX (Ocean Drilling Program), 387-399.

Tiedemann, R., Sarnthein, M., and Shackleton, N.J., 1994. Astronomic timescale for the Pliocene Atlantic $\delta^{18} \mathrm{O}$ and dust flux records of Ocean Drilling Program Site 659. Paleoceanography, 9:619-638.

Van der Zwaan, G.J., and Gudjonsson, L., 1986. Middle Miocene-Pliocene stable isotope stratigraphy and paleoceanography of the Mediterranean. Mar. Micropaleontol., 10:71-90.

Van Os, B.J.H., Lourens, L.J., Hilgen, F.J., de Lange, G.J., and Beaufort, L., 1994. The formation of Pliocene sapropels and carbonate cycles in the Mediterranean: diagenesis, dilution, and productivity. Paleoceanography, 9:601-617.

Vergnaud-Grazzini, C., 1983. Reconstruction of Mediterranean Late Cenozoic hydrography by means of carbon isotope analyses. Utrecht Micropaleontol. Bull., 30:25-47.

Vergnaud-Grazzini, C., Devaux, M., and Znaidi, J., 1986. Stable isotope "anomalies" in Mediterranean Pleistocene records. Mar. Micropaleontol., 10:35-69.

Vergnaud-Grazzini, C., Ryan, W.B.F., and Cita, M.B., 1977. Stable isotope fractionation, climatic change and episodic stagnation in the Eastern Mediterranean during the Late Quaternary. Mar. Micropaleontol., 2:353370.

Vergnaud Grazzini, C., Saliège, J.F., Urrutiaguer, M.J., and Iannace, A. 1990. Oxygen and carbon isotope stratigraphy of ODP Hole 653A and Site 654: the Pliocene-Pleistocene glacial history recorded in the Tyrrhenian Basin (West Mediterranean). In Kastens, K.A., Mascle, J., et al., Proc. ODP, Sci. Results, 107: College Station, TX (Ocean Drilling Program), 361-386.

Williams, D.F., and Thunell, R.C., 1979. Faunal and oxygen isotopic evidence for surface water salinity changes during sapropel formation in the eastern Mediterranean. Sediment. Geol., 23:81-93.

Williams, D.F., Thunell, R.C., and Kennett, J.P., 1978. Periodic freshwater flooding and stagnation of the eastern Mediterranean Sea during the late Quaternary. Science, 201:252-254.

Williams, D.F., Thunell, R.C., Tappa, E.J., Rio, D., and Raffi, I., 1988. Chronology of the Pleistocene oxygen isotope record: 0-1.88 million years before present. Palaeogeogr., Palaeoclimatol., Palaeoecol., 64:221-240.

Wüst, G., 1961. On the vertical circulation of the Mediterranean Sea. J. Geophys. Res., 66:3261-3271.

Zagwin, W.H., 1974. The Plio-Pleistocene boundary in western and southern Europe. Boreas, 3:75-97.

Date of initial receipt: 7 January 1997

Date of acceptance: 2 July 1997

Ms 160SR-014 